\title{
Enhanced Sensitivity in RIACT/MQ-MAS NMR Experiments Using Rotor Assisted Population Transfer
}

\author{
H.-T. Kwak, ${ }^{*}$ S. Prasad, ${ }^{*}$ Z. Yao, ${ }^{*}$ P. J. Grandinetti, ${ }^{* 1}$ J. R. Sachleben, $\dagger$ and L. Emsley $\ddagger$ \\ * Department of Chemistry and $\dagger$ Campus Chemical Instrumentation Center, Ohio State University, Columbus, Ohio 43210-1173; and $\ddagger$ Laboratoire de \\ Stéréochimie et des Interactions Moléculaires, UMR 5532 ENS-Lyon/CNRS, Ecole Normale Supérieure de Lyon, 69364 Lyon, France
}

Received November 29, 2000; revised February 9, 2001; published online April 17, 2001

\begin{abstract}
The rotor assisted population transfer (RAPT) sequence is used to enhance the sensitivity of the RIACT(II) experiment for spin-3/2 quadrupolar nuclei. A detailed theoretical analysis of the polarizations that contribute to different types of MQ-MAS experiments is provided. In particular, two polarization pathways are distinguished for the creation of triple-quantum coherence. The existence of these pathways is experimentally demonstrated by comparing the sensitivities of different sequences with and without RAPT preparation. (c) 2001 Academic Press
\end{abstract}

Key Words: MQ-MAS; RIACT; RAPT; quadrupolar nuclei; solidstate NMR.

\section{INTRODUCTION}

Solid-state NMR of quadrupolar nuclei has undergone a renaissance in the past decade starting with techniques such as double rotation (DOR) (1) and dynamic angle spinning (DAS) (2-4) which provided high-resolution isotropic spectra of quadrupolar nuclei for the first time, followed by the subsequent introduction of the transition correlated magic-angle spinning experiments: multiple-quantum MAS (MQ-MAS) $(5,6)$ and satellite transition MAS (ST-MAS) (7). From a mechanical point of view the transition correlated magic-angle spinning experiments are easier techniques to implement experimentally as they can be performed with most commercial MAS probes and have gained the most widespread use. While all these techniques are welcome additions to the solid-state NMR spectroscopist's toolbox, the inherently low sensitivity of many quadrupolar nuclei still remains an obstacle to their full exploitation.

Although it was understood quite early in the history of NMR that the polarization of the central transition of quadrupolar nuclei can be enhanced by transferring polarization from the satellite transitions (8), attention was only focused on this possibility when Haase and Conradi (9) developed a technique for selective inversion of the outer satellite transitions using frequency swept adiabatic passages to enhance the central transition by a factor of $2 I$ in static samples. Kentgens and Verhagen (10) later employed

1 To whom correspondence should be addressed. E-mail: grandinetti.1@ osu.edu. amplitude-modulated double-frequency adiabatic sweeps to enhance the central transition polarization in samples under both static and MAS conditions. More recently, we devised a simple technique called RAPT (rotor assisted population transfer) (11) where a fast $180^{\circ}$ phase alternating pulse train during magicangle spinning is used to prepare a selectively excited state in which the populations of all eigenstates $|m\rangle$ with the same sign of $m$ are equal, resulting in an enhanced central $m=-1 / 2 \rightarrow 1 / 2$ transition polarization. In general, a theoretical maximum enhancement factor of $I+1 / 2$ can be obtained with this selective "saturation" of the satellite transitions. Although polarization enhancement by selective saturation of the satellite transitions does not provide as much of an enhancement as selective inversion, nevertheless it has the important advantage over selective inversion that it can be performed under sample rotation and obtained for all crystallite orientations simultaneously.

Such enhancement schemes can be easily combined with high-resolution solid-state techniques such as DOR and DAS, since these techniques draw their coherences from the polarization of the central transition. In contrast, understanding the effect of these schemes in combination with MQ-MAS experiments requires an understanding of the polarization source for coherences in MQ-MAS, which will in turn depend on the particular pulse scheme used to prepare the multiple-quantum coherence. For example, in both the traditional high-power single-pulse $(6,12-14)$ scheme and the more recent low-power rotary resonance (FASTER) single-pulse (15) preparation method, the resulting triple-quantum coherence comes mainly from the equilibrium polarization associated with the $m= \pm 3 / 2$ states. In contrast, in the rotationally induced adiabatic coherence transfer (RIACT (II)) scheme (16) the triple-quantum coherence comes from the equilibrium polarization associated with the $m= \pm 1 / 2$ states. Thus, it should be straightforward to apply schemes like RAPT to enhance the sensitivity of RIACT(II) experiments, but RAPT should actually reduce the sensitivity of MQ-MAS sequences that draw their coherences from the equilibrium polarization associated with the $m= \pm 3 / 2$ states.

In this paper we experimentally demonstrate that the application of RAPT before RIACT(II) does indeed improve the sensitivity of the RIACT(II) experiment by a factor of 2 in the 
spin-3/2 system. In addition, we also apply RAPT before a conventional MQ-MAS sequence and demonstrate that the sensitivity is reduced as predicted.

\section{EXPERIMENTAL}

All NMR spectra were acquired at $9.4 \mathrm{~T}(130.93697 \mathrm{MHz}$ ${ }^{87} \mathrm{Rb}$ frequency) with a Bruker DMX 400 spectrometer, using a Bruker 4-mm MAS probehead, capable of achieving a radiofrequency nutation rate $\left(v_{1}\right)$ of $175 \mathrm{kHz}$. A bandpass filter was used in between the probe and the ADC to reduce the reflecting voltage from the probe caused by the high-power RAPT pulse train. The solid-state ${ }^{87} \mathrm{Rb}$ resonance of $\mathrm{RbCl}$ was used to calibrate the radiofrequency field strength and also as the external frequency reference $\left(\delta_{R b C l}=0\right)$. The sample used to demonstrate the utility of the new experiments was polycrystalline $\mathrm{RbClO}_{4}$, which has ${ }^{87} \mathrm{Rb}$ quadrupolar coupling parameters (17) of $C_{q}=3.2 \mathrm{MHz}$ and $\eta_{q}=0.21$. The shifted-echo hypercomplex method (18) was used in acquiring and processing all two-dimensional data. The effective $T_{1}$ of the ${ }^{87} \mathrm{Rb}$ central transition in $\mathrm{RbClO}_{4}$ was measured to be $145 \mathrm{~ms}$ using a saturation recovery experiment. All experiments were performed using a 1-s recycle delay and a spinning speed of $12 \mathrm{kHz}$. The 200-ns delay between each pulse in the $X-\bar{X}$ pulse train of RAPT was used to allow time for the transmitter phase to stabilize. The $X-\bar{X}$ pulse lengths were equal and were optimized experimentally. The inverse of the total time to complete one $X-\bar{X}$ interval (including the 200-ns delays) is defined as the RAPT modulation frequency, $v_{m}$.

\section{THEORY}

To demonstrate how the mechanisms for triple-quantum preparation differ between single-pulse preparation and RIACT(II), we now look at these two mechanisms from a more theoretical perspective. In general, we will consider two possible polarization sources for the triple-quantum coherence, the polarization associated with the $m= \pm 3 / 2$ states and the polarization associated with the $m= \pm 1 / 2$ states. In other words, starting with a density operator of $\rho=\mathbf{I}_{z}=3 \mathbf{I}_{z}^{1-4}+\mathbf{I}_{z}^{2-3}$, and using the single transition operator notation $(19,20)$, we consider two polarization sources for generating triple-quantum coherence,

$$
\begin{gathered}
3 \mathbf{I}_{z}^{1-4} \stackrel{\mathbf{R}_{x}^{1-4}}{\longrightarrow} 3 \mathbf{I}_{y}^{1-4} . \\
\mathbf{I}_{z}^{2-3} \stackrel{\mathbf{R}_{x}^{2-3}}{\longrightarrow} \mathbf{I}_{y}^{1-4} .
\end{gathered}
$$

Because the first rotation utilizes the greater equilibrium polarization of the 1-4 transition, we call it the "greater path" to distinguish it from the second that converts the lesser equilibrium polarization of the $2-3$ transition into $\mathbf{I}_{y}^{1-4}$. While the greater path has the potential to provide three times the triplequantum coherence of the lesser path, it should be noted that the relaxation time needed for the 1-4 transition to return to its equilibrium value may be different than that for the 2-3 transition.

In the discussion that follows we will consider evolution under a Hamiltonian containing the first-order quadrupolar and the radiofrequency interactions:

$$
\tilde{\mathbf{H}} / \hbar=\omega_{q} A_{2,0}\left(\Lambda_{q}\right) \mathbf{T}_{2,0}-\omega_{1} \mathbf{I}_{x},
$$

where $A_{2,0}\left(\Lambda_{q}\right)$ is an element of an irreducible spherical tensor which in its principal axis system (PAS) has the values $\rho_{2,0}=1 / \sqrt{6}$ and $\rho_{2, \pm 2}=\rho_{2,0} \cdot \eta_{q} / \sqrt{6}$. Here $\eta_{q}$ is the quadrupolar coupling asymmetry parameter, and $\Lambda_{q}$ are the Euler angles $(\alpha, \beta, \gamma)$ between the lab frame and the PAS frame. The quadrupolar coupling constant is given by $C_{q}=e^{2} q Q / h$ (or $e^{2} q Q /\left(4 \pi \epsilon_{0} h\right)$ in SI units) and the quadrupolar splitting by $\omega_{q}=6 \pi C_{q} / 2 I(2 I-1)$.

\subsection{Single-Pulse Triple-Quantum Preparation}

On the basis of earlier work on multiple quantum excitation in solids $(19,20)$ it was first assumed in MQMAS articles $(6,12)$ that triple-quantum coherence would be created at a rate proportional to $\omega_{1}^{3} / \omega_{Q}^{2}$ and come from the polarization associated with the 1-4 transition. This assumption was based on a perturbation expansion of the eigenstates and eigenvalues in the limit that $\left|\omega_{1}\right| \ll\left|W_{Q}\left(\Lambda_{q}\right)\right|$ leading to the approximate rotating frame Hamiltonian for a spin $I=3 / 2$ system of

$$
\begin{aligned}
\tilde{\mathbf{H}} / \hbar \approx & g_{-} W_{Q}\left(\Lambda_{q}\right)\left(\mathbf{I}_{z}^{1-2}-\mathbf{I}_{z}^{3-4}\right) \\
& -\left(1-g_{+}\right) \omega_{1} \mathbf{I}_{x}^{1-4}-\left(1+g_{+}\right) \omega_{1} \mathbf{I}_{x}^{2-3}
\end{aligned}
$$

where $W_{Q}\left(\Lambda_{q}\right)=\sqrt{6} \omega_{q} A_{2,0}\left(\Lambda_{q}\right)$ and $g_{ \pm}$is written in a series expansion in the low RF limit as

$$
\begin{aligned}
& g_{-}=1+\frac{3}{2} \frac{\omega_{1}^{2}}{W_{Q}^{2}}+\frac{3}{8} \frac{\omega_{1}^{4}}{W_{Q}^{4}}-\frac{57}{16} \frac{\omega_{1}^{6}}{W_{Q}^{6}}+\frac{867}{128} \frac{\omega_{1}^{8}}{W_{Q}^{8}}+\cdots \\
& g_{+}=1-\frac{3}{2} \frac{\omega_{1}^{2}}{W_{Q}^{2}}+\frac{15}{8} \frac{\omega_{1}^{4}}{W_{Q}^{4}}+\frac{21}{16} \frac{\omega_{1}^{6}}{W_{Q}^{6}}-\frac{1893}{128} \frac{\omega_{1}^{8}}{W_{Q}^{8}}+\cdots
\end{aligned}
$$

The presence of $\mathbf{I}_{x}^{1-4}$ in this approximate Hamiltonian clearly shows that triple-quantum coherence can be created by taking the "greater path," that is, $\mathbf{I}_{z}^{1-4}$ of our equilibrium density operator into $\mathbf{I}_{y}^{1-4}$ at a nutation frequency of $\left(1-g_{+}\right) \omega_{1}$, that is,

$$
\operatorname{Tr}\left\{e^{-(i / \hbar) \tilde{\mathbf{H}} t} \mathbf{I}_{z} e^{(i / \hbar) \tilde{\mathbf{H}} t} \mathbf{I}_{y}^{1-4}\right\}=\frac{3}{2} \sin \left[\left(1-g_{+}\right) \omega_{1} t\right]
$$

In this approximation a zeroth-order expansion of the eigenvectors for this Hamiltonian in the low RF power limit was used without restricting its eigenvalues to obtain the effective rotating frame Hamiltonian (see Appendix A.1). 
Subsequent numerical studies (21), however, showed that in the short pulse limit the triple-quantum oscillations in a polycrystalline sample occurred at a faster rate that is proportional to $\omega_{1}$ and not $\omega_{1}^{3} / \omega_{Q}^{2}$. Clearly, a different subset of crystallite orientations than those with $\left|\omega_{1}\right| \ll\left|W_{Q}\left(\Lambda_{q}\right)\right|$ was contributing more significantly to the total triple-quantum coherence created by a single pulse. Although the $\omega_{1}^{3} / \omega_{Q}^{2}$ assumption is not valid, it does not follow that the polarization source of the triple-quantum coherence is not the $m= \pm 3 / 2$ states. Thus, in order to determine the major polarization source for triplequantum coherence generated by a single pulse in a polycrystalline sample we have performed numerical simulations as a function of quadrupole coupling constant with a constant RF field strength of $\omega_{1} / 2 \pi=100 \mathrm{kHz}$ and sample spinning speed of $\omega_{R} / 2 \pi=10 \mathrm{kHz}$ starting with three different initial density operators of $\rho(0)=\mathbf{I}_{z}^{2-3}, \rho(0)=3 \mathbf{I}_{z}^{1-4}$, and $\rho(0)=\mathbf{I}_{z}$. These results are shown in Fig. 1 for three cases having quadrupolar

(A)

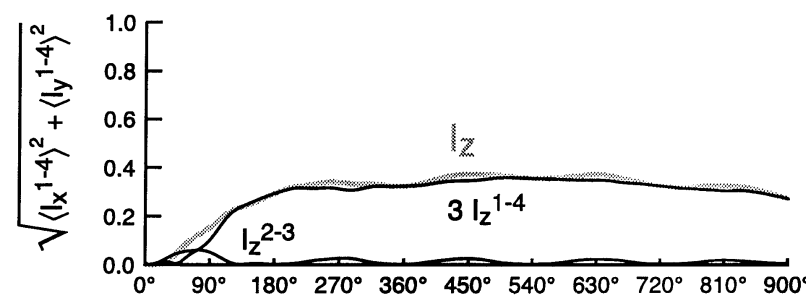

(B)

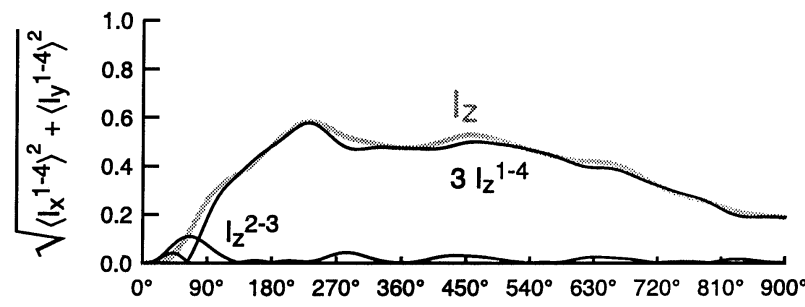

(C)

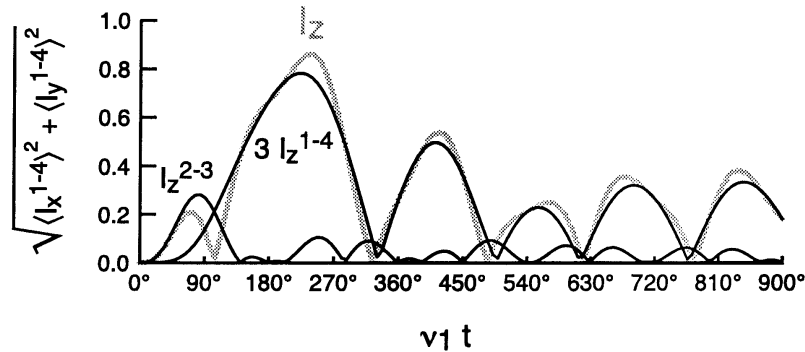

FIG. 1. Creation of triple-quantum coherence starting from the three different initial density matrices, $\rho(0)=\mathbf{I}_{z}^{2-3}$ (solid black line), $\rho(0)=3 \mathbf{I}_{z}^{1-4}$ (solid black line), and $\rho(0)=\mathbf{I}_{z}$ (solid gray line) as a function of pulse duration with $\eta_{q}=0$ and a constant RF field strength of $\omega_{1} / 2 \pi=100 \mathrm{kHz}$ and spinning speed of $\omega_{R} / 2 \pi=10 \mathrm{kHz}$. In (A) $C_{q}=2.5 \mathrm{MHz}$, (B) $C_{q}=1.5 \mathrm{MHz}$, and (C) $C_{q}=0.5 \mathrm{MHz}$. All simulations were based on a full numerical density matrix calculation and were averaged over 3722 crystallite orientations. The continuous motion of the rotor was approximated by discretizing each rotor period into 512 smaller time-independent periods, according to standard methods (22). coupling constants of $C_{q}=0.5,1.5$, and $2.5 \mathrm{MHz}$. In all plots the triple-quantum coherence created from an initial density operator of $\rho(0)=\mathbf{I}_{z}$ is shown as a thick gray line, and the triplequantum coherence created from $\rho(0)=\mathbf{I}_{z}^{2-3}$ and $\rho(0)=3 \mathbf{I}_{z}^{1-4}$ is shown as black lines.

In general, the evolution of the triple-quantum coherence generated from $\rho(0)=\mathbf{I}_{z}$ more closely follows the $\rho(0)=3 \mathbf{I}_{z}^{1-4}$ curve, except at very short pulse lengths where it more closely follows the $\rho(0)=\mathbf{I}_{z}^{2-3}$ curve. Clearly, the most significant triple-quantum intensity is generated at the longer pulse lengths where the majority of the coherence is drawn from the equilibrium polarization associated with the $m= \pm 3 / 2$ states. Thus, assuming that the majority of MQ-MAS experiments would be performed under such optimized conditions, we conclude that the single-pulse triple-quantum preparation in MQ-MAS generally follows the greater path of triple-quantum excitation.

\subsection{RIACT Triple-Quantum Preparation}

While the ideas in the previous section were based on earlier theories of multiple-quantum dynamics in static samples, the ideas employed in RIACT (16) for triple-quantum excitation are based on the theory of spin locking of quadrupolar nuclei under magic-angle spinning as first described by Vega (23). In this section we present a brief overview of this theory as described by Baltisberger et al. (24).

In the case of RF excitation in a rotating sample where the pulse length is not short compared to the rotor period, we transform into the time-dependent diagonal frame and write the propagator in the rotating frame as

$$
\mathbf{U}(t, 0)=\mathbf{V}(t) \cdot \mathbf{T} \cdot e^{-\frac{i}{\hbar} \int_{0}^{t}\left[\mathbf{D}(s)+i \hbar \dot{\mathbf{V}}^{\dagger}(s) \mathbf{V}(s)\right] d s} \cdot \mathbf{V}^{\dagger}(0),
$$

where $\mathbf{T}$ is the Dyson time ordering operator and $\mathbf{V}(t)$ is the transformation that diagonalizes the Hamiltonian at time $t$ according to

$$
\mathbf{D}(t)=\mathbf{V}^{\dagger}(t) \mathbf{H}(t) \mathbf{V}(t)
$$

When $\|\mathbf{D}(t)\| \gg\left\|i \dot{\mathbf{V}}^{\dagger}(t) \mathbf{V}(t)\right\|$ we can apply the adiabatic approximation (in our specific case, there are no diagonal components in $i \dot{\mathbf{V}}^{\dagger}(t) \mathbf{V}(t)$, thus we can neglect this contribution in the adiabatic approximation) and write our propagator as

$$
\mathbf{U}^{(a)}(t, 0)=\mathbf{V}(t) \cdot e^{-\frac{i}{\hbar} \int_{0}^{t} \mathbf{D}(s) d s} \cdot \mathbf{V}^{\dagger}(0) .
$$

Conversely, when $\left\|i \dot{\mathbf{V}}^{\dagger}(t) \mathbf{V}(t)\right\| \gg\|\mathbf{D}(t)\|$ we can apply the sudden approximation and write our propagator as

$$
\mathbf{U}^{(s)}(t, 0)=\mathbf{V}(t) \cdot e^{\int_{0}^{t} \dot{\mathbf{V}}^{\dagger}(s) \mathbf{V}(s) d s} \cdot \mathbf{V}^{\dagger}(0) .
$$

In the case of RF excitation of a second-order broadened 


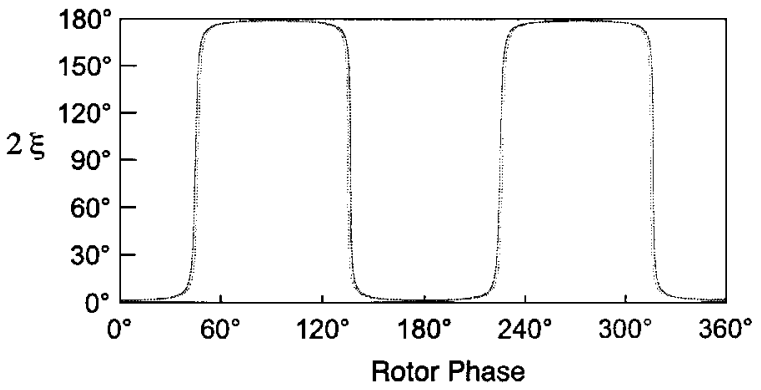

FIG. 2. Plots of $2 \xi^{1-3}(t)$ and $2 \xi^{2-4} t$ as a function of the rotor phase for a crystallite spinning about the magic angle $\left(54.74^{\circ}\right)$ with PAS oriented perpendicular to the rotor, using $C_{q}=2.5 \mathrm{MHz}, \eta_{q}=0.0$.

quadrupolar nucleus under currently available sample spinning speeds, we will find that at any given instant nuclei in nearly all crystallite orientations can be described using the adiabatic approximation, and it is only when $W_{Q}(\Lambda(t))$ passes through zero that we will we need to consider other possibilities.

In the case of a spin $I=3 / 2$ system experiencing the firstorder quadrupolar and RF interactions, we can use the exact analytical diagonalization $(25,19)$ and obtain (see the Appendix)

$$
\mathbf{D}(t)=\frac{\omega_{1}}{2}\left(\mathbf{E}^{1-3}-\mathbf{E}^{2-4}\right)-\omega^{1-3} \mathbf{I}_{z}^{1-3}-\omega^{2-4} \mathbf{I}_{z}^{2-4}
$$

and

$$
i \dot{\mathbf{V}}^{\dagger}(t) \mathbf{V}(t)=2 \dot{\xi}^{2-4}(t) \mathbf{I}_{y}^{2-4}+2 \dot{\xi}^{1-3}(t) \mathbf{I}_{y}^{1-3}
$$

Fig. 2 shows a plot of the values of $2 \xi^{1-3}$ and $2 \xi^{2-4}$, the angles in the 1-3 and 2-4 subspaces, respectively, needed to diagonalize the Hamiltonian, as a function of the rotor phase for those crystallites that undergo the largest excursion of $W_{Q}\left(\Lambda_{q}(t)\right)$ during this period. Note that for a majority of the period $2 \xi^{1-3}$ and $2 \xi^{2-4}$ have values near either 0 or $\pi$.

The size of $i \dot{\mathbf{V}}^{\dagger}(t) \mathbf{V}(t)$ is related to the rate of change of the eigenstates and has the form of Lorenztian impulse functions as follows:

$$
2 \dot{\xi}^{1-3}(t)=\frac{\sqrt{3} \omega_{1} \omega_{Q}}{\left[\omega_{1}-\omega_{Q} A_{2,0}(t)\right]^{2}+3 \omega_{1}^{2}} \cdot \frac{d A_{2,0}(t)}{d t}
$$

and

$$
2 \dot{\xi}^{2-4}(t)=\frac{\sqrt{3} \omega_{1} \omega_{Q}}{\left[\omega_{1}+\omega_{Q} A_{2,0}(t)\right]^{2}+3 \omega_{1}^{2}} \cdot \frac{d A_{2,0}(t)}{d t} .
$$

Plots of $2 \dot{\xi}^{1-3}(t)$ and $2 \dot{\xi}^{2-4}(t)$ as a function of rotor period are shown in Fig. 3. Notice that the $i \dot{\mathbf{V}}^{\dagger}(t) \mathbf{V}(t)$ terms are only nonnegligible near the zero crossings of $W_{Q}(\Lambda(t))$. To indicate whether a zero crossing is in the adiabatic intermediate, or sudden regime, an adiabaticity parameter (24) is defined as

$$
\alpha=-\frac{\omega^{1-3}\left(t_{\text {zero }}\right)}{2 \dot{\xi}^{1-3}\left(t_{\text {zero }}\right)}=\frac{\omega^{2-4}\left(t_{\text {zero }}\right)}{2 \dot{\xi}^{2-4}\left(t_{\text {zero }}\right)}=a\left(\Lambda_{q}^{\prime}, t\right) \cdot \frac{\omega_{1}^{2}}{\omega_{Q} \omega_{R}},
$$

where

$$
\begin{aligned}
\frac{1}{\sqrt{2} a\left(\Lambda_{q}^{\prime}, t\right)} & =\frac{1}{\omega_{R}} \frac{d A_{2,0}(t)}{d t} \\
& =-i \sum_{k^{\prime} \neq 0} k^{\prime} e^{-i k^{\prime}\left(\omega_{R} t+\alpha\right)} d_{k^{\prime}, 0}^{(2)}\left(\beta_{R}\right) A_{2, k^{\prime}}\left(\Lambda_{q}^{\prime}\right) .
\end{aligned}
$$

This definition of the adiabaticity parameter is similar to the one used by Vega (23); however, now there is an additional orientation dependence which comes from the time derivative of $A_{20}(t)$. When the value of $\alpha$ is much larger than, much less than, or on the order of 1 , the zero crossing will be adiabatic, sudden, or intermediate, respectively.

While the sudden approximation does not hold for all times, the terms $2 \dot{\xi}^{1-3}(t)$, and $2 \dot{\xi}^{2-4}(t)$ have the form of an impulse function with an integrated area of $\pi$ centered near the zero crossing of $W_{Q}(\Lambda(t))$. This can result in a rapid transition between the adiabatic propagator of Eq. [2] and the sudden propagator of Eq. [3].
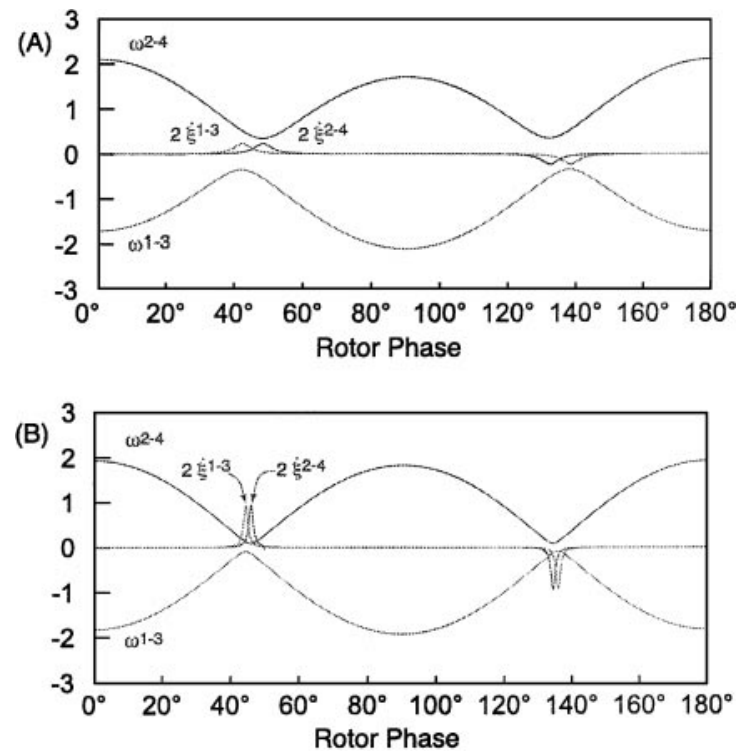

FIG. 3. Plots of diagonal and off-diagonal coefficients $\omega^{1-3}, \omega^{2-4}$, $2 \dot{\xi}^{1-3}(t)$, and $2 \dot{\xi}^{2-4}(t)$ of the time-dependent effective Hamiltonian in Eqs. [4] and [5], as a function of the rotor phase for a crystallite spinning at $\omega_{R} / 2 \pi=12.5 \mathrm{kHz}$ about the magic angle $\left(54.74^{\circ}\right)$ with PAS oriented perpendicular to the rotor and $C_{q}=2.5 \mathrm{MHz}, \eta_{q}=0.0$. In (A) an RF field strength of $\omega_{1} / 2 \pi=200 \mathrm{kHz}$ is used and the adiabatic approximation holds at the zero crossing of the quadrupolar splitting. In (B) an RF field strength of $\omega_{1} / 2 \pi=50 \mathrm{kHz}$ is used and the sudden approximation holds at the zero crossing of the quadrupolar splitting. 
Crystallites which pass through the zero crossing in neither an adiabatic nor a sudden regime fall into the intermediate regime. This type of evolution is the most difficult to calculate analytically. To determine the evolution of the density matrix in the interemediate regime, contributions from both $\mathbf{D}(t)$ and $\dot{\mathbf{V}}^{\dagger}(t) \mathbf{V}(t)$, which do not commute at all times, must be used to construct the propagator. Vega (23) has shown with numerical simulations that spins undergoing an intermediate regime zero crossing evolve into non-spin-locked states and thus result in a significant loss of CP intensity.

3.2.1. RIACT-Adiabatic passage. Using this theoretical framework we now examine the RIACT mechanism (16) for transferring coherence between the triple-quantum and singlequantum central transition states. In the adiabatic approximation we can ignore the term $i \dot{\mathbf{V}}^{\dagger}(t) \mathbf{V}(t)$ at all times and therefore use Eq. [2]. Vega (23) showed that the coherences $\mathbf{I}_{x}^{1-4}$ and $\mathbf{I}_{x}^{2-3}$ will interconvert with every zero crossing of $W_{Q}(\Lambda(t))$ under an RF spin lock in the adiabatic limit. This can be seen using the equation above and propagating $\mathbf{I}_{x}^{2-3}$ under the adiabatic propagator with the RF pulse along $x$ to obtain

$$
\begin{aligned}
\rho(t)= & \mathbf{U}_{x}^{(a)}(t, 0) \cdot \mathbf{I}_{x}^{2-3} \cdot \mathbf{U}_{x}^{(a) \dagger}(t, 0) \\
= & \mathbf{V}_{x}(t) \cdot \exp \left\{-\frac{i}{\hbar} \int_{0}^{t} \mathbf{D}(s) d s\right\} \\
& \cdot \mathbf{V}_{x}^{\dagger}(0) \cdot \mathbf{I}_{x}^{2-3} \cdot \mathbf{V}_{x}(0) \cdot \exp \left\{\frac{i}{\hbar} \int_{0}^{t} \mathbf{D}(s) d s\right\} \cdot \mathbf{V}_{x}^{\dagger}(t) .
\end{aligned}
$$

If we start (arbitrarily) the spin lock at a point where $2 \xi_{1} \approx$ $2 \xi_{2} \approx 0$ (i.e., $\left|W_{Q}\left(\Lambda_{q}\right)\right| \gg\left|\omega_{1}\right|$ and $W_{Q}\left(\Lambda_{q}\right)>0$ ), then the innermost sandwich in our propagator yields

$$
\mathbf{V}_{x}^{\dagger}\left(2 \xi_{1} \approx 2 \xi_{2} \approx 0\right) \cdot \mathbf{I}_{x}^{2-3} \cdot \mathbf{V}_{x}\left(\xi_{1} \approx \xi_{2} \approx 0\right)=\mathbf{I}_{z}^{2-3}
$$

Since $\mathbf{I}_{z}^{2-3}$ commutes with the Hamiltonian in the diagonal frame we have no evolution of $\mathbf{I}_{z}^{2-3}$ due to $\mathbf{U}_{D}(t, 0)=$ $\exp \left\{-(i / \hbar) \int_{0}^{t} \mathbf{D}(s) d s\right\}$. If we turn off the RF spin lock pulse after $W_{Q}\left(\Lambda_{q}\right)$ changes sign due to the rotor motion, then the transformation out of the time-dependent diagonal frame occurs with $2 \xi_{1} \approx 2 \xi_{2} \approx \pi$ (i.e., $\left|W_{Q}\left(\Lambda_{q}\right)\right| \gg\left|\omega_{1}\right|$ and $W_{Q}\left(\Lambda_{q}\right)<0$ ) and we have

$\rho(t)=\mathbf{V}_{x}\left(2 \xi_{1} \approx 2 \xi_{2} \approx \pi\right) \cdot \mathbf{I}_{z}^{2-3} \cdot \mathbf{V}_{x}^{\dagger}\left(2 \xi_{1} \approx 2 \xi_{2} \approx \pi\right)=\mathbf{I}_{x}^{1-4}$

Thus we see that with every zero crossing in $W_{Q}(\Lambda(t))$ the central transition $\mathbf{I}_{x}^{2-3}$ coherence is converted into triple-quantum $\mathbf{I}_{x}^{1-4}$. Similarly, one can also show that with every zero crossing $\mathbf{I}_{x}^{1-4}$ is converted into $\mathbf{I}_{x}^{2-3}$, that is,

$$
\mathbf{I}_{x}^{2-3} \stackrel{\mathbf{U}_{x}^{(a)}(t, 0)}{\longrightarrow} \mathbf{I}_{x}^{1-4} .
$$

Of course, the same behavior is observed if we apply our spin lock on coherences along the $y$ axis, that is,

$$
\mathbf{I}_{y}^{2-3} \stackrel{\mathbf{U}_{y}^{(a)}(t, 0)}{\longrightarrow} \mathbf{I}_{y}^{1-4}
$$

In contrast, all coherences or populations orthogonal to the RF spin lock direction will evolve and will thus lead to rapid dephasing in a polycrystalline or amorphous sample.

This mechanism for interconversion between triple-quantum and central transition coherence with each zero crossing of $W_{Q}$ not only forms the basis of RIACT for MQ-MAS preparation and excitation but also plays an important role during crosspolarization experiments in MAS (26), DAS (24), and MQ-MAS $(27-30)$.

\subsection{The RAPT Effect}

Finally, we note that the effect of the RAPT sequence is to prepare the initial density operator in a state that enhances the polarization of the central $(m= \pm 1 / 2)$ transition at the expense of triple-quantum $(m= \pm 3 / 2)$ polarization,

$$
\rho=3 \mathbf{I}_{z}^{1-4}+\mathbf{I}_{z}^{2-3} \stackrel{R A P T}{\longrightarrow} 2 \mathbf{I}_{z}^{1-4}+2 \mathbf{I}_{z}^{2-3},
$$

the impact of this rearrangement being that the sensitivity of experiments like RIACT which draw their triple-quantum coherence from the central transition will be enhanced by a factor of 2, while those like the conventional single-pulse MQ-MAS experiment will be diminished by a factor of $2 / 3$.

\section{RESULTS AND DISCUSSION}

In light of the above discussion we investigated the effect of a RAPT preparation sequence optimized for the central transition of ${ }^{87} \mathrm{Rb}$ in $\mathrm{RbClO}_{4}$ on the sensitivity of the RIACT(II) MQMAS experiment, as well as on the conventional single-pulse excitation and mixing MQ-MAS experiment.

Using the RAPT enhanced RIACT(II) sequence, which was constructed by simply placing the RAPT preparation sequence (11) in front of the RIACT(II) sequence (16) as shown in Fig. 4A, an experimental enhancement factor of 1.8 was obtained. The spectrum of Fig. 4A was obtained with an RF field strength of $170 \mathrm{kHz}$, a modulation frequency $\left(v_{m}\right)$ of $695 \mathrm{kHz}$, and a RAPT pulse train duration of $90.7 \mu \mathrm{s}$, which is close to one rotor period, $\tau_{r}=83.3 \mu \mathrm{s}$. The length of the selective central transition excitation pulse was $0.75 \mu \mathrm{s}$, and the spin-lock pulse was $20.83 \mu \mathrm{s}$, which is $\tau_{r} / 4$, as specified by Wu et al. (16). The interval $\tau$ between the RAPT preparation was increased to approximately $0.4 \mathrm{~ms}$ to act as a $z$-filter, eliminating all transverse coherences and the need to modify the RIACT(II) phase cycle.

The experimental enhancement factor of 1.8 is very close to the theoretical value of $I+1 / 2=2$, showing the good agreement with theory. Moreover, it is particularly important to 
notice that the anisotropic MQ-MAS lineshape observed using the RAPT enhancement is undistorted with respect to the conventional RIACT(II) experiment, indicating that all crystallites in the sample are affected in the same way by the RAPT preparation. The anisotropic cross sections are shown in Fig. 4B for the RIACT(II) experiment with RAPT preparation (shown as a solid line) and without RIACT(II) preparation (shown as a dashed line). The lack of distortion in the enhanced lineshape is one of the key advantages of this method.

To ensure that our theoretical understanding outlined in the previous section is correct, the effect of the RAPT preparation on the conventional MQ-MAS experiment was also investigated by placing the RAPT pulse train before the conventional MQMAS pulse sequence, as shown in Fig. 5A. For this experiment, the RF field strength used for the RAPT preparation and the conventional MQ-MAS sequence sequence was $170 \mathrm{kHz}$. The experimentally optimized excitation pulse length was $6 \mu \mathrm{s}$, and the conversion pulse length was $5.5 \mu \mathrm{s}$.

In this case we see from the anisotropic lineshapes of Fig. 5B an experimental reduction in signal using the RAPT preparation by a factor of 0.6 , which is in close agreement with the factor of $2 / 3$ predicted by theory in the previous section. Thus, these experimental demonstrations tend to strongly support our
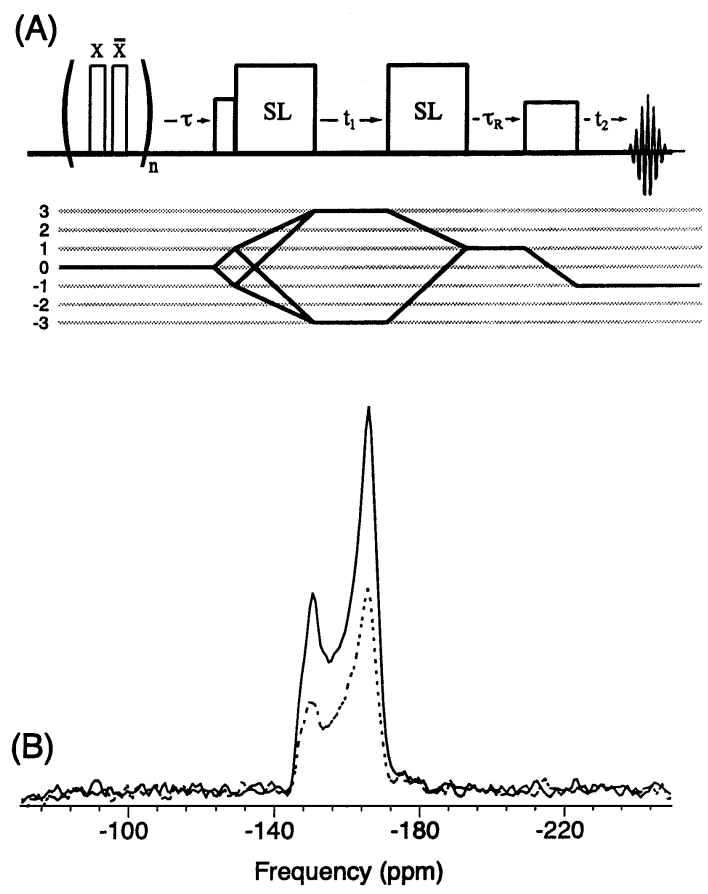

FIG. 4. (A) RIACT(II) pulse sequence with RAPT preparation. (B) A comparison of the anisotropic projections from the $\omega_{2}$ dimension of the ${ }^{87} \mathrm{Rb}$ RIACT(II) experiment in polycrystalline $\mathrm{RbClO}_{4}$ with (solid line) and without (dashed line) the RAPT preparation. A total of $64 t_{1}$ points with $15-\mu$ s increments were acquired with $\tau$ set to 20 rotor periods and the spinning rate to $12 \mathrm{kHz}$. The spectrum was zero filled once in the $t_{2}$ and $t_{1}$ domains. A factor of 1.8 sensitivity enhancement was achieved by applying RAPT preparation before RIACT(II).
(A)
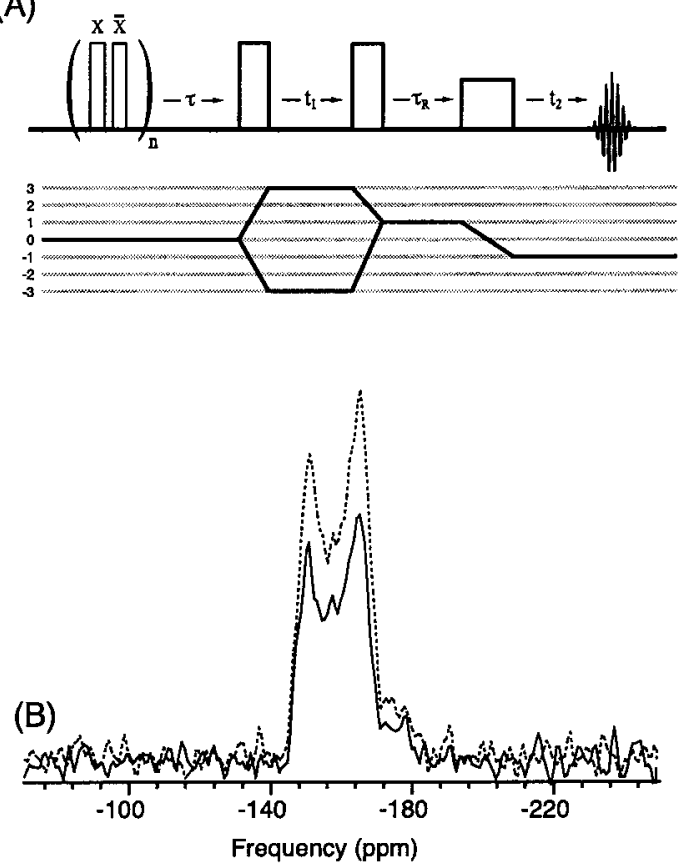

FIG. 5. (A) MQ-MAS pulse sequence with RAPT preparation. (B) A comparison of the anisotropic projections from the $\omega_{2}$ dimension of the ${ }^{87} \mathrm{Rb}$ MQ-MAS experiment in polycrystalline $\mathrm{RbClO}_{4}$ with (solid line) and without (dashed line) the RAPT preparation. A total of $64 t_{1}$ points with $15-\mu \mathrm{s}$ increments were acquired with $\tau$ set to 20 rotor periods and the spinning rate to $12 \mathrm{kHz}$. The spectrum was zero filled once in the $t_{2}$ and $t_{1}$ domains. In this example the experimental sensitivity is diminished by a factor of 0.6 in MQ-MAS using the RAPT preparation.

theoretical interpretation of the mechanisms of these experiments in terms of the greater and lesser paths.

\section{CONCLUSION}

We have provided a detailed analysis of the polarizations that contribute to different types of MQ-MAS experiments. We distinguish two preparation pathways to create triplequantum coherence, the first from outer transition polarization and the second from inner transition polarization. These pathways are dubbed the greater and lesser paths, respectively. We find that "conventional" single-pulse preparation MQ-MAS sequences principally benefit from the greater path, while sequences of the RIACT type utilize predominantly the lesser path.

In light of this discussion we have proposed the combination of the RAPT preparation sequence with RIACT(II) and we achieve an experimental factor of 1.8 sensitivity enhancement without significant changes in the RIACT(II) anisotropic lineshape.

In contrast we also demonstrate that, as predicted, the application of the RAPT pulse train in front of conventional MQ-MAS leads to a factor of 0.6 sensitivity reduction. 


\section{APPENDIX}

\section{A.1. Diaginalization of the $I=3 / 2$ Rotating Frame Hamiltonian During RF Irradiation}

In this Appendix we examine the diagonalization of the rotating frame Hamiltonian for a quadrupolar coupled $I=3 / 2$ during RF irradiation. Our starting Hamiltonian is

$$
\tilde{\mathbf{H}} / \hbar=\omega_{q} A_{2,0}\left(\Lambda_{q}\right) \mathbf{T}_{2,0}-\omega_{1} \mathbf{I}_{x} .
$$

For $I=3 / 2$, this Hamiltonian can be written in terms of fictitious spin half operators $(19,20,31)$

$\mathbf{H} / \hbar=W_{Q}\left(\Lambda_{q}\right)\left(\mathbf{I}_{z}^{1-2}-\mathbf{I}_{z}^{3-4}\right)-\sqrt{3} \omega_{1}\left(\mathbf{I}_{x}^{1-2}+\mathbf{I}_{x}^{3-4}\right)-2 \omega_{1} \mathbf{I}_{x}^{2-3}$,

where $W_{Q}\left(\Lambda_{q}\right)=\sqrt{6} \omega_{q} A_{2,0}\left(\Lambda_{q}\right)$. This Hamiltonian can be diagonalized $(19,25)$ using the transformation

$$
\mathbf{V}\left(2 \xi^{1-3}, 2 \xi^{2-4}\right)=e^{i \frac{\pi}{2} \mathbf{I}_{y}^{1-4}} e^{-i \frac{\pi}{2} \mathbf{I}_{y}^{2-3}} e^{2 i \xi^{1-3} \mathbf{I}_{y}^{1-3}} e^{2 i \xi^{2-4} \mathbf{I}_{y}^{2-4}},
$$

with

$$
\begin{aligned}
& \tan \left(2 \xi^{1-3}\right)=\frac{-\sqrt{3} \omega_{1}}{W_{Q}\left(\Lambda_{q}\right)-\omega_{1}}, \\
& \tan \left(2 \xi^{2-4}\right)=\frac{-\sqrt{3} \omega_{1}}{W_{Q}\left(\Lambda_{q}\right)+\omega_{1}} .
\end{aligned}
$$

In this diagonal frame we have

$$
\begin{aligned}
\mathbf{D}=\mathbf{V}^{\dagger} \mathbf{H V}= & \frac{1}{2} f_{-} W_{Q}\left(\Lambda_{q}\right)\left(\mathbf{E}^{1-4}-\mathbf{E}^{2-3}\right) \\
& +\omega_{1}\left[\left(1-f_{+}\right) \mathbf{I}_{z}^{1-4}-\left(1+f_{+}\right) \mathbf{I}_{z}^{2-3}\right],
\end{aligned}
$$

where

$$
\begin{aligned}
& f_{-}=\frac{\omega^{2-4}\left(\omega_{1}\right)-\omega^{1-3}\left(\omega_{1}\right)}{2 W_{Q}\left(\Lambda_{q}\right)} \\
& f_{+}=\frac{\omega^{2-4}\left(\omega_{1}\right)+\omega^{1-3}\left(\omega_{1}\right)}{2 \omega_{1}}
\end{aligned}
$$

or, written in terms of double-quantum operators,

$$
\mathbf{D}=\frac{1}{2} \omega_{1}\left(\mathbf{E}^{1-3}-\mathbf{E}^{2-4}\right)-\omega^{1-3} \mathbf{I}_{z}^{1-3}-\omega^{2-4} \mathbf{I}_{z}^{2-4},
$$

where

$$
\begin{aligned}
\omega^{1-3} & =-\sqrt{3 \omega_{1}^{2}+\left(W_{Q}\left(\Lambda_{q}\right)-\omega_{1}\right)^{2}} \\
& =-\left(f_{-} W_{Q}\left(\Lambda_{q}\right)-f_{+} \omega_{1}\right), \\
\omega^{2-4} & =\sqrt{3 \omega_{1}^{2}+\left(W_{Q}\left(\Lambda_{q}\right)+\omega_{1}\right)^{2}}=f_{-} W_{Q}\left(\Lambda_{q}\right)+f_{+} \omega_{1} .
\end{aligned}
$$

\section{A.1.1. Perturbation Expansion in the Low $\omega_{1}$ Limit}

The analytical solution given above can be used to obtain a series expansion about the low RF power limit (i.e., about $\left.\omega_{1}=0\right)$ for the eigenvectors to obtain

$$
\begin{aligned}
\cos 2 \xi^{1-3}\left(\omega_{1}\right)= & \frac{W_{Q}\left(\Lambda_{q}\right)}{\sqrt{W_{Q}\left(\Lambda_{q}\right)^{2}}}\left[1+0-\frac{3}{2} \frac{\omega_{1}^{2}}{W_{Q}\left(\Lambda_{q}\right)^{2}}\right. \\
& -\frac{3 \omega_{1}^{3}}{W_{Q}\left(\Lambda_{q}\right)^{3}}-\frac{9}{8} \frac{\omega_{1}^{4}}{W_{Q}\left(\Lambda_{q}\right)^{4}} \\
& \left.+\frac{15}{2} \frac{\omega_{1}^{5}}{W_{Q}\left(\Lambda_{q}\right)^{5}}+\cdots\right], \\
\sin 2 \xi^{1-3}\left(\omega_{1}\right)= & \frac{W_{Q}\left(\Lambda_{q}\right)}{\sqrt{W_{Q}\left(\Lambda_{q}\right)^{2}}\left[0-\frac{\sqrt{3} \omega_{1}}{W_{Q}\left(\Lambda_{q}\right)}-\frac{\sqrt{3} \omega_{1}^{2}}{W_{Q}\left(\Lambda_{q}\right)^{2}}\right.} \\
& +\frac{\sqrt{3}}{2} \frac{\omega_{1}^{3}}{W_{Q}\left(\Lambda_{q}\right)^{3}}+\frac{7 \sqrt{3}}{2} \frac{\omega_{1}^{4}}{W_{Q}\left(\Lambda_{q}\right)^{4}} \\
& \left.+\frac{37 \sqrt{3}}{8} \frac{\omega_{1}^{5}}{W_{Q}\left(\Lambda_{q}\right)^{5}}+\cdots\right],
\end{aligned}
$$

and

$$
\begin{aligned}
\cos 2 \xi^{2-4}\left(\omega_{1}\right)= & \frac{W_{Q}\left(\Lambda_{q}\right)}{\sqrt{W_{Q}\left(\Lambda_{q}\right)^{2}}}\left[1+0-\frac{3}{2} \frac{\omega_{1}^{2}}{W_{Q}\left(\Lambda_{q}\right)^{2}}\right. \\
& +\frac{3 \omega_{1}^{3}}{W_{Q}\left(\Lambda_{q}\right)^{3}}-\frac{9}{8} \frac{\omega_{1}^{4}}{W_{Q}\left(\Lambda_{q}\right)^{4}} \\
& \left.-\frac{15}{2} \frac{\omega_{1}^{5}}{W_{Q}\left(\Lambda_{q}\right)^{5}}+\cdots\right], \\
\sin 2 \xi^{2-4}\left(\omega_{1}\right)= & \frac{W_{Q}\left(\Lambda_{q}\right)}{\sqrt{W_{Q}\left(\Lambda_{q}\right)^{2}}}\left[0-\frac{\sqrt{3} \omega_{1}}{W_{Q}\left(\Lambda_{q}\right)}+\frac{\sqrt{3} \omega_{1}^{2}}{W_{Q}\left(\Lambda_{q}\right)^{2}}\right. \\
& +\frac{\sqrt{3}}{2} \frac{\omega_{1}^{3}}{W_{Q}\left(\Lambda_{q}\right)^{3}}-\frac{7 \sqrt{3}}{2} \frac{\omega_{1}^{4}}{W_{Q}\left(\Lambda_{q}\right)^{4}} \\
& \left.+\frac{37 \sqrt{3}}{8} \frac{\omega_{1}^{5}}{W_{Q}\left(\Lambda_{q}\right)^{5}}+\cdots\right] .
\end{aligned}
$$

and for the the eigenvalues we obtain

$$
f_{ \pm}=\frac{W_{Q}}{\sqrt{W_{Q}^{2}}} g_{ \pm},
$$

where

$$
\begin{aligned}
& g_{-}=1+\frac{3}{2} \frac{\omega_{1}^{2}}{W_{Q}^{2}}+\frac{3}{8} \frac{\omega_{1}^{4}}{W_{Q}^{4}}-\frac{57}{16} \frac{\omega_{1}^{6}}{W_{Q}^{6}}+\frac{867}{128} \frac{\omega_{1}^{8}}{W_{Q}^{8}}+\cdots \\
& g_{+}=1-\frac{3}{2} \frac{\omega_{1}^{2}}{W_{Q}^{2}}+\frac{15}{8} \frac{\omega_{1}^{4}}{W_{Q}^{4}}+\frac{21}{16} \frac{\omega_{1}^{6}}{W_{Q}^{6}}-\frac{1893}{128} \frac{\omega_{1}^{8}}{W_{Q}^{8}}+\cdots
\end{aligned}
$$


An important simplification afforded by these expansions is that coherence transfer efficiency during a pulse is determined entirely by the eigenvectors, while the nutation frequency is determined entirely by the eigenvalues. Therefore, we are not required to use expansions of equal order for both the eigenvalues, $f_{ \pm}$, and the eigenvectors, $2 \xi^{1-3}$ and $2 \xi^{2-4}$, in order to obtain accurate predictions for the transfer efficiency and nutation frequency during the preparation and mixing periods.

To first order the angles $2 \xi^{1-3}$ and $2 \xi^{2-4}$ are equal, and to zeroth order they are equal to either zero or $\pi$ depending on the sign of $W_{Q}$. When $W_{Q}>0$ we transform our diagonal Hamiltonian back to the rotating frame using $2 \xi^{1-3}=2 \xi^{2-4}=0$, and when $W_{Q}<0$ we transform our diagonal Hamiltonian back to the rotating frame using $2 \xi^{1-3}=2 \xi^{2-4}=\pi$. In both cases the final result is

$\mathbf{H} \approx g_{-} W_{Q}\left(\mathbf{I}_{z}^{1-2}-\mathbf{I}_{z}^{3-4}\right)-\left(1-g_{+}\right) \omega_{1} \mathbf{I}_{x}^{1-4}-\left(1+g_{+}\right) \omega_{1} \mathbf{I}_{x}^{2-3}$.

\section{A.2. Operator Transformations Between Rotating and Diagonal Frame}

\section{A.2.1. Transformations from the Rotating Frame into the}

Diagonal Frame for the Spin I =3/2 Case

$$
\begin{aligned}
\mathbf{V}_{ \pm}^{\dagger} \mathbf{I}_{ \pm}^{1-4} \mathbf{V}= & \frac{1}{2}\left(\mathbf{I}_{ \pm}^{1-4}-\mathbf{I}_{\mp}^{1-4}\right) \cos \xi^{2-4} \cos \xi^{1-3} \\
& +\frac{1}{2}\left(\mathbf{I}_{ \pm}^{2-3}-\mathbf{I}_{\mp}^{2-3}\right) \sin \xi^{2-4} \sin \xi^{1-3} \\
& -\frac{1}{2}\left(\mathbf{I}_{ \pm}^{1-2}-\mathbf{I}_{\mp}^{1-2}\right) \sin \xi^{2-4} \cos \xi^{1-3} \\
& +\frac{1}{2}\left(\mathbf{I}_{ \pm}^{3-4}-\mathbf{I}_{\mp}^{3-4}\right) \cos \xi^{2-4} \sin \xi^{1-3} \\
& -\frac{1}{2} \mathbf{I}_{x}^{2-4} \sin 2 \xi^{2-4}-\frac{1}{2} \mathbf{I}_{x}^{1-3} \sin 2 \xi^{1-3} \\
& -\mathbf{I}_{z}^{1-4} \cos ^{2} \xi^{1-3} \cos ^{2} \xi^{2-4} \\
& +\mathbf{I}_{z}^{2-3} \sin ^{2} \xi^{1-3} \sin ^{2} \xi^{2-4} \\
& -\mathbf{I}_{z}^{1-2} \cos ^{2} \xi^{1-3} \sin ^{2} \xi^{2-4} \\
& -\mathbf{I}_{z}^{3-4} \sin ^{2} \xi^{1-3} \cos ^{2} \xi^{2-4} \\
\mathbf{V}_{ \pm}^{\dagger} \mathbf{I}_{ \pm}^{2-3} \mathbf{V}= & \frac{1}{2}\left(\mathbf{I}_{ \pm}^{1-4}-\mathbf{I}_{\mp}^{1-4}\right) \sin \xi^{2-4} \sin \xi^{1-3} \\
& +\frac{1}{2}\left(\mathbf{I}_{ \pm}^{2-3}-\mathbf{I}_{\mp}^{2-3}\right) \cos \xi^{2-4} \cos \xi^{1-3} \\
& +\frac{1}{2}\left(\mathbf{I}_{ \pm}^{1-2}-\mathbf{I}_{\mp}^{1-2}\right) \cos \xi^{2-4} \sin \xi^{1-3} \\
& -\frac{1}{2}\left(\mathbf{I}_{ \pm}^{3-4}-\mathbf{I}_{\mp}^{3-4}\right) \sin ^{2-4} \cos \xi^{1-3} \\
& +\frac{1}{2} \mathbf{I}_{x}^{2-4} \sin ^{2-\xi^{2-4}} \\
&
\end{aligned}
$$

$$
\begin{aligned}
& +\frac{1}{2} \mathbf{I}_{x}^{1-3} \sin 2 \xi^{1-3}+\mathbf{I}^{2-3} \cos ^{2} \xi^{1-3} \cos ^{2} \xi^{2-4} \\
& -\mathbf{I}_{z}^{1-4} \sin ^{2} \xi^{1-3} \sin ^{2} \xi^{2-4} \\
& -\mathbf{I}_{z}^{1-2} \sin ^{2} \xi^{1-3} \cos ^{2} \xi^{2-4} \\
& -\mathbf{I}_{z}^{3-4} \cos ^{2} \xi^{1-3} \sin ^{2} \xi^{2-4} \\
\mathbf{V}^{\dagger} \mathbf{I}_{z}^{2-3} \mathbf{V}=- & \frac{1}{2}\left(\mathbf{I}_{+}^{2-3}+\mathbf{I}_{-}^{2-3}\right) \cos \xi^{2-4} \cos \xi^{1-3} \\
& +\frac{1}{2}\left(\mathbf{I}_{+}^{1-4}+\mathbf{I}_{-}^{1-4}\right) \sin \xi^{2-4} \sin \xi^{1-3} \\
& +\frac{1}{2}\left(\mathbf{I}_{+}^{1-2}+\mathbf{I}_{-}^{1-2}\right) \cos \xi^{2-4} \sin \xi^{1-3} \\
& -\frac{1}{2}\left(\mathbf{I}_{+}^{3-4}+\mathbf{I}_{-}^{3-4}\right) \sin \xi^{2-4} \cos \xi^{1-3} \\
\mathbf{V}^{\dagger} \mathbf{I}_{z}^{1-4} \mathbf{V}=- & \frac{1}{2}\left(\mathbf{I}_{+}^{1-4}+\mathbf{I}_{-}^{1-4}\right) \cos \xi^{2-4} \cos \xi^{1-3} \\
& -\frac{1}{2}\left(\mathbf{I}_{+}^{2-3}+\mathbf{I}_{-}^{2-3}\right) \sin \xi^{2-4} \sin \xi^{1-3} \\
& -\frac{1}{2}\left(\mathbf{I}_{+}^{1-2}+\mathbf{I}_{-}^{1-2}\right) \cos \xi^{2-4} \sin \xi^{1-3} \\
& +\frac{1}{2}\left(\mathbf{I}_{+}^{3-4}+\mathbf{I}_{-}^{3-4}\right) \sin \xi^{2-4} \cos \xi^{1-3}
\end{aligned}
$$

\section{A.2.2. Transformations from the Diagonal Frame into the} Rotating Frame for the Spin I=3/2 Case

$$
\begin{aligned}
& \mathbf{V} \mathbf{I}_{+}^{2-3} \mathbf{V}^{\dagger} \\
& =\frac{1}{2}\left(\mathbf{I}_{+}^{2-3}-\mathbf{I}_{-}^{2-3}\right) \cos \xi^{1-3} \cos \xi^{2-4}-\mathbf{I}_{z}^{2-3} \cos \xi^{1-3} \cos \xi^{2-4} \\
& \quad+\frac{1}{2}\left(\mathbf{I}_{+}^{1-4}-\mathbf{I}_{-}^{1-4}\right) \sin \xi^{1-3} \sin \xi^{2-4}-\mathbf{I}_{z}^{1-4} \sin \xi^{1-3} \sin \xi^{2-4} \\
& \quad+\frac{1}{2}\left(\mathbf{I}_{+}^{1-2} \cos \xi^{1-3} \sin \xi^{2-4}+\mathbf{I}_{-}^{1-2} \sin \xi^{1-3} \cos \xi^{2-4}\right) \\
& -\frac{1}{2}\left(\mathbf{I}_{+}^{3-4}+\sin \xi^{1-3} \cos \xi^{2-4}+\mathbf{I}_{-}^{3-4} \cos \xi^{1-3} \sin \xi^{2-4}\right) \\
& -\frac{1}{2}\left(\mathbf{I}_{+}^{2-4} \sin \xi^{1-3} \cos \xi^{2-4}-\mathbf{I}_{-}^{2-4} \cos \xi^{1-3} \sin \xi^{2-4}\right) \\
& -\frac{1}{2}\left(\mathbf{I}_{+}^{1-3} \cos \xi^{1-3} \sin \xi^{2-4}-\mathbf{I}_{-}^{1-3} \sin \xi^{1-3} \cos \xi^{2-4}\right) \\
& \mathbf{V I}_{-}^{2-3} \mathbf{V}^{\dagger}=-\frac{1}{2}\left(\mathbf{I}_{+}^{2-3}-\mathbf{I}_{-}^{2-3}\right) \cos \xi^{1-3} \cos \xi^{2-4} \\
& \quad-\mathbf{I}_{z}^{2-3} \cos \xi^{1-3} \cos \xi^{2-4} \\
& \quad-\frac{1}{2}\left(\mathbf{I}_{+}^{1-4}-\mathbf{I}_{-}^{1-4}\right) \sin \xi^{1-3} \sin \xi^{2-4} \\
& \quad-\mathbf{I}_{z}^{1-4} \sin \xi^{1-3} \sin \xi^{2-4}+\frac{1}{2}\left(\mathbf{I}_{+}^{1-2} \sin \xi^{1-3} \cos \xi^{2-4}\right.
\end{aligned}
$$




$$
\begin{aligned}
& \left.+\mathbf{I}_{-}^{1-2} \cos \xi^{1-3} \sin \xi^{2-4}\right)-\frac{1}{2}\left(\mathbf{I}_{+}^{3-4} \cos \xi^{1-3} \sin \xi^{2-4}\right. \\
& \left.+\mathbf{I}_{-}^{3-4} \sin \xi^{1-3} \cos \xi^{2-4}\right)+\frac{1}{2}\left(\mathbf{I}_{+}^{2-4} \cos \xi^{1-3} \sin \xi^{2-4}\right. \\
& \left.-\mathbf{I}_{-}^{2-4} \sin \xi^{1-3} \cos \xi^{2-4}\right)+\frac{1}{2}\left(\mathbf{I}_{+}^{1-3} \sin \xi^{1-3} \cos \xi^{2-4}\right. \\
& \left.-\mathbf{I}_{-}^{1-3} \cos \xi^{1-3} \sin \xi^{2-4}\right)
\end{aligned}
$$

$$
\begin{aligned}
\mathbf{V} \mathbf{I}_{z}^{2-3} & \mathbf{V}^{\dagger} \\
= & \frac{1}{4}\left(\mathbf{I}_{+}^{2-3}+\mathbf{I}_{-}^{2-3}\right)\left(\cos ^{2} \xi^{1-3}+\cos ^{2} \xi^{2-4}\right) \\
& +\frac{1}{2} \mathbf{E}^{2-3}\left(\cos ^{2} \xi^{2-4}-\cos ^{2} \xi^{1-3}\right) \\
& +\frac{1}{4}\left(\mathbf{I}_{+}^{1-4}+\mathbf{I}_{-}^{1-4}\right)\left(\sin ^{2} \xi^{1-3}+\sin ^{2} \xi^{2-4}\right) \\
& +\mathbf{E}^{1-4}\left(\sin ^{2} \xi^{2-4}-\sin ^{2} \xi^{1-3}\right) \\
& +\frac{1}{4}\left(\mathbf{I}_{+}^{1-2}+\mathbf{I}_{-}^{1-2}\right)\left(\cos \xi^{1-3} \sin \xi^{1-3}-\cos \xi^{2-4} \sin \xi^{2-4}\right) \\
& +\frac{1}{4}\left(\mathbf{I}_{+}^{3-4}+\mathbf{I}_{-}^{3-4}\right)\left(\cos \xi^{1-3} \sin \xi^{1-3}-\cos \xi^{2-4} \sin \xi^{2-4}\right) \\
& -\frac{1}{4}\left(\mathbf{I}_{+}^{2-4}+\mathbf{I}_{-}^{2-4}\right)\left(\cos \xi^{1-3} \sin \xi^{1-3}+\cos \xi^{2-4} \sin \xi^{2-4}\right) \\
& -\frac{1}{4}\left(\mathbf{I}_{+}^{1-3}+\mathbf{I}_{-}^{1-3}\right)\left(\cos \xi^{1-3} \sin \xi^{1-3}+\cos \xi^{2-4} \sin \xi^{2-4}\right)
\end{aligned}
$$

$$
\begin{aligned}
\mathbf{V I} \mathbf{I}_{+}^{1-4} & \mathbf{V}^{\dagger} \\
= & \frac{1}{2}\left(\mathbf{I}_{+}^{1-4}-\mathbf{I}_{-}^{1-4}\right) \cos \xi^{1-3} \cos \xi^{2-4} \\
& +\mathbf{I}^{1-4} \cos \xi^{1-3} \cos \xi^{2-4} \\
& +\frac{1}{2}\left(\mathbf{I}_{+}^{2-3}-\mathbf{I}_{-}^{2-3}\right) \sin \xi^{1-3} \sin \xi^{2-4}+\mathbf{I}^{2-3} \sin \xi^{1-3} \sin \xi^{2-4} \\
& +\frac{1}{2}\left(\mathbf{I}_{+}^{1-2} \cos \xi^{1-3} \sin \xi^{2-4}+\mathbf{I}_{-}^{1-2} \sin \xi^{1-3} \cos \xi^{2-4}\right) \\
& -\frac{1}{2}\left(\mathbf{I}_{+}^{3-4} \sin \xi^{1-3} \cos \xi^{2-4}+\mathbf{I}_{+}^{3-4} \cos \xi^{1-3} \sin \xi^{2-4}\right) \\
& +\frac{1}{2}\left(\mathbf{I}_{+}^{2-4} \sin \xi^{1-3} \cos \xi^{2-4}-\mathbf{I}_{-}^{2-4} \cos \xi^{1-3} \sin \xi^{2-4}\right) \\
& +\frac{1}{2}\left(\mathbf{I}_{+}^{1-3} \cos \xi^{1-3} \sin \xi^{2-4}-\mathbf{I}_{-}^{1-3} \sin \xi^{1-3} \cos \xi^{2-4}\right)
\end{aligned}
$$

$$
\begin{aligned}
\mathbf{V I}_{-}^{1-4} \mathbf{V}^{\dagger} & \\
= & -\frac{1}{2}\left(\mathbf{I}_{+}^{1-4}-\mathbf{I}_{-}^{1-4}\right) \cos \xi^{1-3} \cos \xi^{2-4} \\
& +\mathbf{I}_{z}^{1-4} \cos \xi^{1-3} \cos \xi^{2-4}-\frac{1}{2}\left(\mathbf{I}_{+}^{2-3}\right.
\end{aligned}
$$

$$
\begin{aligned}
& \left.-\mathbf{I}_{-}^{2-3}\right) \sin \xi^{1-3} \sin \xi^{2-4}+\mathbf{I}_{z}^{2-3} \sin \xi^{1-3} \sin \xi^{2-4} \\
& +\frac{1}{2}\left(\mathbf{I}_{+}^{1-2} \sin \xi^{1-3} \cos \xi^{2-4}+\mathbf{I}_{-}^{1-2} \cos \xi^{1-3} \sin \xi^{2-4}\right) \\
& -\frac{1}{2}\left(\mathbf{I}_{+}^{3-4} \cos \xi^{1-3} \sin \xi^{2-4}+\mathbf{I}_{+}^{3-4} \sin \xi^{1-3} \cos \xi^{2-4}\right) \\
& -\frac{1}{2}\left(\mathbf{I}_{+}^{2-4} \cos \xi^{1-3} \sin \xi^{2-4}-\mathbf{I}_{-}^{2-4} \sin \xi^{1-3} \cos \xi^{2-4}\right) \\
& -\frac{1}{2}\left(\mathbf{I}_{+}^{1-3} \sin \xi^{1-3} \cos \xi^{2-4}-\mathbf{I}_{-}^{1-3} \cos \xi^{1-3} \sin \xi^{2-4}\right)
\end{aligned}
$$

$$
\begin{aligned}
\mathbf{V} \mathbf{I}_{z}^{1-4} & \mathbf{V}^{\dagger} \\
= & -\frac{1}{4}\left(\mathbf{I}_{+}^{1-4}+\mathbf{I}_{-}^{1-4}\right)\left(\cos ^{2} \xi^{1-3}+\cos ^{2} \xi^{2-4}\right) \\
& -\frac{1}{2} \mathbf{E}^{1-4}\left(\cos ^{2} \xi^{2-4}-\cos ^{2} \xi^{1-3}\right) \\
& -\frac{1}{4}\left(\mathbf{I}_{+}^{2-3}+\mathbf{I}_{-}^{2-3}\right)\left(\sin ^{2} \xi^{1-3}+\sin ^{2} \xi^{2-4}\right) \\
& -\mathbf{E}^{2-3}\left(\sin ^{2} \xi^{2-4}-\sin ^{2} \xi^{1-3}\right) \\
& +\frac{1}{4}\left(\mathbf{I}_{+}^{1-2}+\mathbf{I}_{-}^{1-2}\right)\left(\cos \xi^{1-3} \sin \xi^{1-3}-\cos \xi^{2-4} \sin \xi^{2-4}\right) \\
& +\frac{1}{4}\left(\mathbf{I}_{+}^{3-4}+\mathbf{I}_{-}^{3-4}\right)\left(\cos \xi^{1-3} \sin \xi^{1-3}-\cos \xi^{2-4} \sin \xi^{2-4}\right) \\
& -\frac{1}{4}\left(\mathbf{I}_{+}^{2-4}+\mathbf{I}_{-}^{2-4}\right)\left(\cos \xi^{1-3} \sin \xi^{1-3}+\cos \xi^{2-4} \sin \xi^{2-4}\right) \\
& -\frac{1}{4}\left(\mathbf{I}_{+}^{1-3}+\mathbf{I}_{-}^{1-3}\right)\left(\cos \xi^{1-3} \sin \xi^{1-3}+\cos \xi^{2-4} \sin \xi^{2-4}\right)
\end{aligned}
$$

\section{ACKNOWLEDGMENTS}

This work was supported in part by a grant from the National Science Foundation (CHE-9807498) and the U.S. Department of Energy. We thank Marek Pruski for helpful discussions. PJG also acknowledges support from the ENS Lyon for a visiting professorship during the early part of this work.

\section{REFERENCES}

1. A. Samoson, E. Lippmaa, and A. Pines, High resolution solid-state NMR. Averaging of second-order effects by means of a double-rotor, Mol. Phys. 65, 1013 (1988).

2. A. Llor and J. Virlet, Towards high-resolution NMR of more nuclei in solids: Sample spinning with time-dependent spinner axis angle, Chem. Phys. Lett. 152, 248-253 (1988).

3. B. F. Chmelka, K. T. Mueller, A. Pines, J. Stebbins, Y. Wu, and J. W. Zwanziger, Oxygen-17 NMR in solids by dynamic-angle spinning and double rotation, Nature 339, 42-43 (1989).

4. K. T. Mueller, G. C. Chingas, and A. Pines, NMR probe for dynamic-angle spinning, Rev. Sci. Instrum. 62, 1445 (1991).

5. L. Frydman and J. S. Harwood, Isotropic spectra of half-integer quadrupolar spins from bidimensional magic-angle spinning NMR, J. Am. Chem. Soc. 117, 5367-5369 (1995). 
6. A. Medek, J. S. Harwood, and L. Frydman, Multiple-quantum magic-angle spinning NMR: A new method for the study of quadrupolar nuclei in solids, J. Am. Chem. Soc. 117, 12779-12787 (1995).

7. Z. Gan, Isotropic NMR spectra of half-integer quadrupolar nuclei using satellite transitions and magic-angle spinning, J. Am. Chem. Soc. 122, 3242 3243 (2000).

8. R. V. Pound, Nuclear electric quadrupole interactions in crystals, Phys. Rev. 79, 685-702 (1950).

9. J. Haase and M. S. Conradi, Sensitivity enhancement for NMR of the central transition of quadrupolar nuclei, Chem. Phys. Lett. 209(3), 287-291 (1993).

10. A. P. M. Kentgens and R. Verhagen, Advantages of double frequency sweeps in static, MAS, and MQMAS NMR of spin $I=3 / 2$ nuclei, Chem. Phys. Lett. 300, 435-443 (1999).

11. Z. Yao, H.-T. Kwak, D. Sakellariou, L. Emsley, and P. J. Grandinetti, Sensitivity enhancement of the central transition NMR signal of quadrupolar nuclei under magic-angle spinning, Chem. Phys. Lett. 327, 85-90 (2000).

12. D. Massiot, B. Touzo, D. Trumeau, J. P. Coutures, J. Virlet, P. Florian, and P. J. Grandinetti, Two-dimensional magic-angle spinning isotropic reconstruction sequences for quadrupolar nuclei, Solid State NMR 6, 73-83 (1996).

13. C. Fernandez and J. P. Amoureux, Triple-quantum MAS-NMR of quadrupolar nuclei, Solid State NMR 5, 315-321 (1996).

14. G. Wu, D. Rovnyak, B. Sun, and R. G. Griffin, High-resolution multiple quantum MAS NMR spectroscopy of half-integer quadrupolar nuclei, Chem. Phys. Lett. 249, 210-217 (1996).

15. T. Vosegaard, P. Florian, D. Massiot, and P. J. Grandinetti, Multiple quantum magic-angle spinning using rotary resonance excitation, J. Chem. Phys. 114, 4620-4624 (2001).

16. G. Wu, D. Rovnyak, and R. G. Griffin, Quantitative multiple-quantum magic-angle spinning NMR spectroscopy of quadrupolar nuclei in solids, J. Am. Chem. Soc. 118, 9326-9332 (1996).

17. T. Vosegaard, J. Skibsted, H. Bildsoe, and H. J. Jakobsen, Quadrupole coupling and anisotropic shielding from single-crystal NMR of the central transition for quadrupolar nuclei. ${ }^{87} \mathrm{Rb} \mathrm{NMR}$ of $\mathrm{RbClO}_{4}$ and $\mathrm{Rb}_{2} \mathrm{SO}_{4}$, J. Magn. Reson. 122, 111-119 (1996).
18. P. J. Grandinetti, J. H. Baltisberger, A. Llor, Y. K. Lee, U. Werner, M. A. Eastman, and A. Pines, Pure absorption-mode lineshapes and sensitivity in two-dimensional dyanmic angle spinning NMR, J. Magn. Reson. A 103, 72-81 (1993).

19. A. Wokaun and R. R. Ernst, Selective excitation and detection in multilevel spin systems: Application of single transition operators, J. Chem. Phys. 67, 1752-1758 (1977).

20. S. Vega, Fictitious spin-1/2 operator formalism for multiple quantum NMR, J. Chem. Phys. 68, 5518-5527 (1978).

21. J. P. Amoureux, C. Fernandez, and L. Frydman, Optimized multiplequantum magic-angle spinning NMR experiments on half-integer quadrupoles, Chem. Phys. Lett. 259, 347-355 (1996).

22. P. Hodgkinson and L. Emsley, Numerical simulation of solid-state nmr experiments, Prog. Nucl. Magn. Reson. Spectrosc. 36(3), 201-239 (2000).

23. A. J. Vega, MAS NMR spin locking of half-integer quadrupolar nuclei, J. Magn. Reson. 96, 50-68 (1992).

24. J. H. Baltisberger, S. L. Gann, P. J. Grandinetti, and A. Pines, Crosspolarization dynamic-angle spinning nuclear magnetic resonance of quadrupolar nuclei, Mol. Phys. 81, 1109-1124 (1994).

25. S. Vega and Y. Naor, Triple quantum NMR on spin systems with $I=3 / 2$ in solids, J. Chem. Phys. 75, 75 (1981).

26. A. J. Vega, CP/MAS of quadrupolar $S=3 / 2$ nuclei, Solid State NMR 1, 17 (1992).

27. C. Fernandez, L. Delevoye, J. P. Amoureux, D. P. Lang, and M. Pruski, ${ }^{27} \mathrm{Al}\left\{{ }^{1} \mathrm{H}\right\}$ cross polarization triple-quantum magic-angle spinning NMR, J. Am. Chem. Soc. 119, 6858-6862 (1997).

28. M. Pruski, D. P. Lang, C. Fernandez, and J. P. Amoureux, Multiple-quantum magic-angle spinning NMR with cross-polarization: Spectral editing of high-resolution spectra of quadrupolar nuclei, Solid State NMR 7, 327331 (1997)

29. S. E. Ashbrook, S. P. Brown, and S. Wimperis, Multiple-quantum crosspolarization in MAS NMR of quadrupolar nuclei, Chem. Phys. Lett. 288, 509-517 (1998)

30. K. H. Lim and C. P. Grey, ${ }^{19} \mathrm{~F} /{ }^{23} \mathrm{Na}$ multiple quantum cross polarization NMR in solids, J. Chem. Phys. 112(17), 7490-7504 (2000).

31. S. Vega and A. Pines, Operator formalism for double quantum NMR, J. Chem. Phys. 66(12), 5624-5644 (1977). 


\section{ERRATUM}

Volume 150, Number 1 (2001), in article "Enhanced Sensitivity in RIACT/MQMAS NMR Experiments Using Rotor Assisted Population Transfer," by H.-T. Kwak, S. Prasad, Z. Yao, P. J. Grandinetti, J. R. Sachleben, and L. Emsley, pages 71-80 (doi: 10.1006/jmre.2001.2313): On page 78, the equations in Appendix A.2.1 should read

$$
\begin{aligned}
\mathbf{V}^{\dagger} \mathbf{I}_{ \pm}^{1-4} \mathbf{V}= & -\mathbf{I}_{z}^{1-4} \cos ^{2} \xi^{2-4}+\mathbf{I}_{z}^{2-3} \sin ^{2} \xi^{1-3}+\mathbf{I}_{z}^{1-2}\left(\cos ^{2} \xi^{2-4}-\cos ^{2} \xi^{1-3}\right) \\
& -\mathbf{I}_{x}^{1-3} \cos \xi^{1-3} \sin \xi^{1-3}-\mathbf{I}_{x}^{2-4} \cos \xi^{2-4} \sin \xi^{2-4} \pm i \mathbf{I}_{y}^{1-4} \cos \xi^{1-3} \cos \xi^{2-4} \\
& \mp i \mathbf{I}_{y}^{1-2} \cos \xi^{1-3} \sin \xi^{2-4} \pm i \mathbf{I}_{y}^{3-4} \sin \xi^{1-3} \cos \xi^{2-4} \pm i \mathbf{I}_{y}^{2-3} \sin \xi^{1-3} \sin \xi^{2-4} \\
\mathbf{V}^{\dagger} \mathbf{I}_{ \pm}^{2-3} \mathbf{V}= & \mathbf{I}_{z}^{2-3} \cos ^{2} \xi^{2-4}-\mathbf{I}_{z}^{1-4} \sin ^{2} \xi^{1-3}+\mathbf{I}_{z}^{3-4}\left(\cos ^{2} \xi^{2-4}-\cos ^{2} \xi^{1-3}\right) \\
& +\mathbf{I}_{x}^{1-3} \cos \xi^{1-3} \sin \xi^{1-3}+\mathbf{I}_{x}^{2-4} \cos \xi^{2-4} \sin \xi^{2-4} \pm i \mathbf{I}_{y}^{2-3} \cos \xi^{1-3} \cos \xi^{2-4} \\
& \pm i \mathbf{I}_{y}^{1-2} \sin \xi^{1-3} \cos \xi^{2-4} \mp i \mathbf{I}_{y}^{3-4} \cos \xi^{1-3} \sin \xi^{2-4} \pm i \mathbf{I}_{y}^{1-4} \sin \xi^{1-3} \sin \xi^{2-4} \\
\mathbf{V}^{\dagger} \mathbf{I}_{z}^{1-4} \mathbf{V}= & \mathbf{I}_{x}^{1-4} \cos \xi^{1-3} \cos \xi^{2-4}-\mathbf{I}_{x}^{2-3} \sin \xi^{1-3} \sin \xi^{2-4} \\
& -\mathbf{I}_{x}^{1-2} \cos \xi^{1-3} \sin \xi^{2-4}+\mathbf{I}_{x}^{3-4} \sin \xi^{1-3} \cos \xi^{2-4} \\
\mathbf{V}^{\dagger} \mathbf{I}_{z}^{2-3} \mathbf{V}= & -\mathbf{I}_{x}^{2-3} \cos \xi^{1-3} \cos \xi^{2-4}+\mathbf{I}_{x}^{1-4} \sin \xi^{1-3} \sin \xi^{2-4} \\
& +\mathbf{I}_{x}^{1-2} \sin \xi^{1-3} \cos \xi^{2-4}-\mathbf{I}_{x}^{3-4} \cos \xi^{1-3} \sin \xi^{2-4}
\end{aligned}
$$

instead of

$$
\begin{aligned}
& \mathbf{V}_{ \pm}^{\dagger} \mathbf{I}_{ \pm}^{1-4} \mathbf{V}= \frac{1}{2}\left(\mathbf{I}_{ \pm}^{1-4}-\mathbf{I}_{\mp}^{1-4}\right) \cos \xi^{2-4} \cos \xi^{1-3}+\frac{1}{2}\left(\mathbf{I}_{ \pm}^{2-3}-\mathbf{I}_{\mp}^{2-3}\right) \sin \xi^{2-4} \sin \xi^{1-3} \\
&-\frac{1}{2}\left(\mathbf{I}_{ \pm}^{1-2}-\mathbf{I}_{\mp}^{1-2}\right) \sin \xi^{2-4} \cos \xi^{1-3}+\frac{1}{2}\left(\mathbf{I}_{ \pm}^{3-4}-\mathbf{I}_{\mp}^{3-4}\right) \cos \xi^{2-4} \sin \xi^{1-3} \\
&-\frac{1}{2} \mathbf{I}_{x}^{2-4} \sin 2 \xi^{2-4}-\frac{1}{2} \mathbf{I}_{x}^{1-3} \sin 2 \xi^{1-3}-\mathbf{I}_{z}^{1-4} \cos ^{2} \xi^{1-3} \cos ^{2} \xi^{2-4} \\
&+\mathbf{I}_{z}^{2-3} \sin ^{2} \xi^{1-3} \sin ^{2} \xi^{2-4}-\mathbf{I}_{z}^{1-2} \cos ^{2} \xi^{1-3} \sin ^{2} \xi^{2-4}-\mathbf{I}_{z}^{3-4} \sin ^{2} \xi^{1-3} \cos ^{2} \xi^{2-4} \\
& \mathbf{V}_{ \pm}^{\dagger} \mathbf{I}_{ \pm}^{2-3} \mathbf{V}= \frac{1}{2}\left(\mathbf{I}_{ \pm}^{1-4}-\mathbf{I}_{\mp}^{1-4}\right) \sin \xi^{2-4} \sin \xi^{1-3}+\frac{1}{2}\left(\mathbf{I}_{ \pm}^{2-3}-\mathbf{I}_{\mp}^{2-3}\right) \cos \xi^{2-4} \cos \xi^{1-3} \\
&+\frac{1}{2}\left(\mathbf{I}_{ \pm}^{1-2}+\mathbf{I}_{\mp}^{1-2}\right) \cos \xi^{2-4} \sin \xi^{1-3}-\frac{1}{2}\left(\mathbf{I}_{ \pm}^{3-4}-\mathbf{I}_{\mp}^{3-4}\right) \sin \xi^{2-4} \cos \xi^{1-3} \\
&+\frac{1}{2} \mathbf{I}_{x}^{2-4} \sin _{2 \xi^{2-4}}+\frac{1}{2} \mathbf{I}_{x}^{1-3} \sin 2 \xi^{1-3}+\mathbf{I}_{z}^{2-3} \cos ^{2} \xi^{1-3} \cos ^{2} \xi^{2-4} \\
&-\mathbf{I}_{z}^{1-4} \sin ^{2} \xi^{1-3} \sin ^{2} \xi^{2-4}-\mathbf{I}_{z}^{1-2} \sin ^{2} \xi^{1-3} \cos ^{2} \xi^{2-4}-\mathbf{I}_{z}^{3-4} \cos ^{2} \xi^{1-3} \sin ^{2} \xi^{2-4} \\
&-\frac{1}{2}\left(\mathbf{I}_{+}^{2-3}+\mathbf{I}_{-}^{2-3}\right) \cos \xi^{2-4} \cos \xi^{1-3}+\frac{1}{2}\left(\mathbf{I}_{+}^{1-4}+\mathbf{I}_{-}^{1-4}\right) \sin \xi^{2-4} \sin ^{1-3} \\
&+\frac{1}{2}\left(\mathbf{I}_{+}^{1-2}+\mathbf{I}_{-}^{1-2}\right) \cos \xi^{2-4} \sin \xi^{1-3}-\frac{1}{2}\left(\mathbf{I}_{+}^{3-4}+\mathbf{I}_{-}^{3-4}\right) \sin \xi^{2-4} \cos \xi^{1-3} \\
& \mathbf{V}^{\dagger} \mathbf{I}_{z}^{2-3} \mathbf{V}
\end{aligned}
$$




$$
\begin{aligned}
\mathbf{V}^{\dagger} \mathbf{I}_{z}^{1-4} \mathbf{V}= & \frac{1}{2}\left(\mathbf{I}_{+}^{1-4}+\mathbf{I}_{-}^{1-4}\right) \cos \xi^{2-4} \cos \xi^{1-3}-\frac{1}{2}\left(\mathbf{I}_{+}^{2-3}+\mathbf{I}_{-}^{2-3}\right) \sin \xi^{2-4} \sin \xi^{1-3} \\
& -\frac{1}{2}\left(\mathbf{I}_{+}^{1-2}+\mathbf{I}_{-}^{1-2}\right) \cos \xi^{2-4} \sin \xi^{1-3}+\frac{1}{2}\left(\mathbf{I}_{+}^{3-4}+\mathbf{I}_{-}^{3-4}\right) \sin \xi^{2-4} \cos \xi^{1-3}
\end{aligned}
$$

On pages 78 and 79, the equations in Appendix A.2.2 should read:

$$
\begin{aligned}
& \mathbf{V} \mathbf{I}_{ \pm}^{2-3} \mathbf{V}^{\dagger}=-\left(\mathbf{I}_{z}^{2-3} \mp i \mathbf{I}_{y}^{2-3}\right) \cos \xi^{1-3} \cos \xi^{2-4}-\left(\mathbf{I}_{z}^{1-4} \mp i \mathbf{I}_{y}^{1-4}\right) \sin \xi^{1-3} \sin \xi^{2-4} \\
& +\frac{1}{2}\left(\mathbf{I}_{ \pm}^{1-2}-\mathbf{I}_{\mp}^{3-4}\right) \cos \xi^{1-3} \sin \xi^{2-4}-\frac{1}{2}\left(\mathbf{I}_{ \pm}^{1-3}-\mathbf{I}_{\mp}^{2-4}\right) \cos \xi^{1-3} \sin \xi^{2-4} \\
& +\frac{1}{2}\left(\mathbf{I}_{\mp}^{1-2}-\mathbf{I}_{ \pm}^{3-4}\right) \sin \xi^{1-3} \cos \xi^{2-4}+\frac{1}{2}\left(\mathbf{I}_{ \pm}^{1-3}-\mathbf{I}_{ \pm}^{2-4}\right) \sin \xi^{1-3} \cos \xi^{2-4} \\
& \mathbf{V} \mathbf{I}_{z}^{2-3} \mathbf{V}^{\dagger}=\mathbf{I}_{x}^{1-4}+\frac{1}{2}\left(\mathbf{I}_{x}^{2-3}-\mathbf{I}_{x}^{1-4}\right)\left(\cos ^{2} \xi^{1-3}+\cos ^{2} \xi^{2-4}\right) \\
& +\frac{1}{2}\left(\mathbf{I}_{x}^{1-2}+\mathbf{I}_{x}^{3-4}\right) \cos \xi^{1-3} \sin \xi^{1-3}-\frac{1}{2}\left(\mathbf{I}_{x}^{1-2}+\mathbf{I}_{x}^{3-4}\right) \cos \xi^{2-4} \sin \xi^{2-4} \\
& +\frac{1}{2}\left(\mathbf{I}_{z}^{1-2}-\mathbf{I}_{z}^{3-4}\right)\left(\cos ^{2} \xi^{1-3}-\cos ^{2} \xi^{2-4}\right)-\frac{1}{2}\left(\mathbf{I}_{x}^{1-3}+\mathbf{I}_{x}^{2-4}\right) \cos \xi^{1-3} \sin \xi^{1-3} \\
& -\frac{1}{2}\left(\mathbf{I}_{x}^{1-3}+\mathbf{I}_{x}^{2-4}\right) \cos \xi^{2-4} \sin \xi^{2-4} \\
& \mathbf{V} \mathbf{I}_{ \pm}^{1-4} \mathbf{V}^{\dagger}=\left(\mathbf{I}_{z}^{1-4} \pm i \mathbf{I}_{y}^{1-4}\right) \cos \xi^{1-3} \cos \xi^{2-4}+\left(\mathbf{I}_{z}^{2-3} \pm i \mathbf{I}_{y}^{2-3}\right) \sin \xi^{1-3} \sin \xi^{2-4} \\
& +\frac{1}{2}\left(\mathbf{I}_{ \pm}^{1-2}-\mathbf{I}_{\mp}^{3-4}\right) \cos \xi^{1-3} \sin \xi^{2-4}+\frac{1}{2}\left(\mathbf{I}_{ \pm}^{1-3}-\mathbf{I}_{\mp}^{2-4}\right) \cos \xi^{1-3} \sin \xi^{2-4} \\
& +\frac{1}{2}\left(\mathbf{I}_{\mp}^{1-2}-\mathbf{I}_{ \pm}^{3-4}\right) \sin \xi^{1-3} \cos \xi^{2-4}-\frac{1}{2}\left(\mathbf{I}_{\mp}^{1-3}-\mathbf{I}_{\mp}^{2-4}\right) \sin \xi^{1-3} \cos \xi^{2-4} \\
& \mathbf{V} \mathbf{I}_{z}^{1-4} \mathbf{V}^{\dagger}=-\mathbf{I}_{x}^{2-3}-\frac{1}{2}\left(\mathbf{I}_{x}^{1-4}-\mathbf{I}_{x}^{2-3}\right)\left(\cos ^{2} \xi^{1-3}+\cos ^{2} \xi^{2-4}\right) \\
& +\frac{1}{2}\left(\mathbf{I}_{x}^{1-2}+\mathbf{I}_{x}^{3-4}\right) \cos \xi^{1-3} \sin \xi^{1-3}-\frac{1}{2}\left(\mathbf{I}_{x}^{1-2}+\mathbf{I}_{x}^{3-4}\right) \cos \xi^{2-4} \sin \xi^{2-4} \\
& +\frac{1}{2}\left(\mathbf{I}_{z}^{1-2}-\mathbf{I}_{z}^{3-4}\right)\left(\cos ^{2} \xi^{1-3}-\cos ^{2} \xi^{2-4}\right)-\frac{1}{2}\left(\mathbf{I}_{x}^{1-3}+\mathbf{I}_{x}^{2-4}\right) \cos \xi^{1-3} \sin \xi^{1-3} \\
& -\frac{1}{2}\left(\mathbf{I}_{x}^{1-3}+\mathbf{I}_{x}^{2-4}\right) \cos \xi^{2-4} \sin \xi^{2-4}
\end{aligned}
$$

instead of

$$
\begin{aligned}
\mathbf{V} \mathbf{I}_{+}^{2-3} \mathbf{V}^{\dagger}= & \frac{1}{2}\left(\mathbf{I}_{+}^{2-3}-\mathbf{I}_{-}^{2-3}\right) \cos \xi^{1-3} \cos \xi^{2-4}-\mathbf{I}_{z}^{2-3} \cos \xi^{1-3} \cos \xi^{2-4} \\
& +\frac{1}{2}\left(\mathbf{I}_{+}^{1-4}-\mathbf{I}_{-}^{1-4}\right) \sin \xi^{1-3} \sin \xi^{2-4}-\mathbf{I}_{z}^{1-4} \sin \xi^{1-3} \sin \xi^{2-4} \\
& +\frac{1}{2}\left(\mathbf{I}_{+}^{1-2} \cos \xi^{1-3} \sin \xi^{2-4}+\mathbf{I}_{-}^{1-2} \sin \xi^{1-3} \cos \xi^{2-4}\right) \\
& -\frac{1}{2}\left(\mathbf{I}_{+}^{3-4} \sin \xi^{1-3} \cos \xi^{2-4}+\mathbf{I}_{-}^{3-4} \cos \xi^{1-3} \sin \xi^{2-4}\right) \\
& -\frac{1}{2}\left(\mathbf{I}_{+}^{2-4} \sin \xi^{1-3} \cos \xi^{2-4}-\mathbf{I}_{-}^{2-4} \cos \xi^{1-3} \sin \xi^{2-4}\right) \\
& -\frac{1}{2}\left(\mathbf{I}_{+}^{1-3}-\cos \xi^{1-3} \sin \xi^{2-4}-\mathbf{I}_{-}^{1-3} \sin \xi^{1-3} \cos \xi^{2-4}\right)
\end{aligned}
$$




$$
\begin{aligned}
& \mathbf{V} \mathbf{I}_{-}^{2-3} \mathbf{V}^{\dagger}=-\frac{1}{2}\left(\mathbf{I}_{+}^{2-3}-\mathbf{I}_{-}^{2-3}\right) \cos \xi^{1-3} \cos \xi^{2-4}-\mathbf{I}_{z}^{2-3} \cos \xi^{1-3} \cos \xi^{2-4} \\
& -\frac{1}{2}\left(\mathbf{I}_{+}^{1-4}-\mathbf{I}_{-}^{1-4}\right) \sin \xi^{1-3} \sin \xi^{2-4}-\mathbf{I}_{z}^{1-4} \sin \xi^{1-3} \sin \xi^{2-4} \\
& +\frac{1}{2}\left(\mathbf{I}_{+}^{1-2} \sin \xi^{1-3} \cos \xi^{2-4}+\mathbf{I}_{-}^{1-2} \cos \xi^{1-3} \sin \xi^{2-4}\right)-\frac{1}{2}\left(\mathbf{I}_{+}^{3-4} \cos \xi^{1-3} \sin \xi^{2-4}\right. \\
& \left.+\mathbf{I}_{-}^{3-4} \sin \xi^{1-3} \cos \xi^{2-4}\right)+\frac{1}{2}\left(\mathbf{I}_{+}^{2-4} \cos \xi^{1-3} \sin \xi^{2-4}-\mathbf{I}_{-}^{2-4} \sin \xi^{1-3} \cos \xi^{2-4}\right) \\
& +\frac{1}{2}\left(\mathbf{I}_{+}^{1-3} \sin \xi^{1-3} \cos \xi^{2-4}-\mathbf{I}_{-}^{1-3} \cos \xi^{1-3} \sin \xi^{2-4}\right) \\
& \mathbf{V I}_{z}^{2-3} \mathbf{V}^{\dagger}=\frac{1}{4}\left(\mathbf{I}_{+}^{2-3}+\mathbf{I}_{-}^{2-3}\right)\left(\cos ^{2} \xi^{1-3}+\cos ^{2} \xi^{2-4}\right)+\frac{1}{2} \mathbf{E}^{2-3}\left(\cos ^{2} \xi^{2-4}-\cos ^{2} \xi^{1-3}\right) \\
& +\frac{1}{4}\left(\mathbf{I}_{+}^{1-4}+\mathbf{I}_{-}^{1-4}\right)\left(\sin ^{2} \xi^{1-3}+\sin ^{2} \xi^{2-4}\right)+\mathbf{E}^{1-4}\left(\sin ^{2} \xi^{2-4}-\sin ^{2} \xi^{1-3}\right) \\
& +\frac{1}{4}\left(\mathbf{I}_{+}^{1-2}+\mathbf{I}_{-}^{1-2}\right)\left(\cos \xi^{1-3} \sin \xi^{1-3}-\cos \xi^{2-4} \sin \xi^{2-4}\right) \\
& \left.+\frac{1}{4} \mathbf{I}_{+}^{3-4}+\mathbf{I}_{-}^{3-4}\right)\left(\cos \xi^{1-3} \sin \xi^{1-3}-\cos \xi^{2-4} \sin \xi^{2-4}\right) \\
& -\frac{1}{4}\left(\mathbf{I}_{+}^{2-4}+\mathbf{I}_{-}^{2-4}\right)\left(\cos \xi^{1-3} \sin \xi^{1-3}+\cos \xi^{2-4} \sin \xi^{2-4}\right) \\
& -\frac{1}{4}\left(\mathbf{I}_{+}^{1-3}+\mathbf{I}_{-}^{1-3}\right)\left(\cos \xi^{1-3} \sin \xi^{1-3}+\cos \xi^{2-4} \sin \xi^{2-4}\right) \\
& \mathbf{V I}_{+}^{1-4} \mathbf{V}^{\dagger}=\frac{1}{2}\left(\mathbf{I}_{+}^{1-4}-\mathbf{I}_{-}^{1-4}\right) \cos \xi^{1-3} \cos \xi^{2-4}+\mathbf{I}_{z}^{1-4} \cos \xi^{1-3} \cos \xi^{2-4} \\
& +\frac{1}{2}\left(\mathbf{I}_{+}^{2-3}-\mathbf{I}_{-}^{2-3}\right) \sin \xi^{1-3} \sin \xi^{2-4}+\mathbf{I}_{z}^{2-3} \sin \xi^{1-3} \sin \xi^{2-4} \\
& +\frac{1}{2}\left(\mathbf{I}_{+}^{1-2} \cos \xi^{1-3} \sin \xi^{2-4}+\mathbf{I}_{-}^{1-2} \sin \xi^{1-3} \cos \xi^{2-4}\right)-\frac{1}{2}\left(\mathbf{I}_{+}^{3-4} \sin \xi^{1-3} \cos \xi^{2-4}\right. \\
& \left.+\mathbf{I}_{-}^{3-4} \cos \xi^{1-3} \sin \xi^{2-4}\right)+\frac{1}{2}\left(\mathbf{I}_{+}^{2-4} \sin \xi^{1-3} \cos \xi^{2-4}-\mathbf{I}_{-}^{2-4} \cos \xi^{1-3} \sin \xi^{2-4}\right) \\
& +\frac{1}{2}\left(\mathbf{I}_{+}^{1-3} \cos \xi^{1-3} \sin \xi^{2-4}-\mathbf{I}_{-}^{1-3} \sin \xi^{1-3} \cos \xi^{2-4}\right) \\
& \mathbf{V I} \mathbf{I}_{-}^{1-4} \mathbf{V}^{\dagger}=-\frac{1}{2}\left(\mathbf{I}_{+}^{1-4}-\mathbf{I}_{-}^{1-4}\right) \cos \xi^{1-3} \cos \xi^{2-4}+\mathbf{I}_{z}^{1-4} \cos \xi^{1-3} \cos \xi^{2-4} \\
& -\frac{1}{2}\left(\mathbf{I}_{+}^{2-3}-\mathbf{I}_{-}^{2-3}\right) \sin \xi^{1-3} \sin \xi^{2-4}+\mathbf{I}_{z}^{2-3} \sin \xi^{1-3} \sin \xi^{2-4} \\
& +\frac{1}{2}\left(\mathbf{I}_{+}^{1-2} \sin \xi^{1-3} \cos \xi^{2-4}+\mathbf{I}_{-}^{1-2} \cos \xi^{1-3} \sin \xi^{2-4}\right)-\frac{1}{2}\left(\mathbf{I}_{+}^{3-4} \cos \xi^{1-3} \sin \xi^{2-4}\right. \\
& \left.+\mathbf{I}_{-}^{3-4} \sin \xi^{1-3} \cos \xi^{2-4}\right)-\frac{1}{2}\left(\mathbf{I}_{+}^{2-4} \cos \xi^{1-3} \sin \xi^{2-4}-\mathbf{I}_{-}^{2-4} \sin \xi^{1-3} \cos \xi^{2-4}\right) \\
& -\frac{1}{2}\left(\mathbf{I}_{+}^{1-3} \sin \xi^{1-3} \cos \xi^{2-4}-\mathbf{I}_{-}^{1-3} \cos \xi^{1-3} \sin \xi^{2-4}\right) \\
& \mathbf{V} \mathbf{I}_{z}^{1-4} \mathbf{V}^{\dagger}=-\frac{1}{4}\left(\mathbf{I}_{+}^{1-4}+\mathbf{I}_{-}^{1-4}\right)\left(\cos ^{2} \xi^{1-3}+\cos ^{2} \xi^{2-4}\right)-\frac{1}{2} \mathbf{E}^{1-4}\left(\cos ^{2} \xi^{2-4}-\cos ^{2} \xi^{1-3}\right) \\
& -\frac{1}{4}\left(\mathbf{I}_{+}^{2-3}+\mathbf{I}_{-}^{2-3}\right)\left(\sin ^{2} \xi^{1-3}+\sin ^{2} \xi^{2-4}\right)-\mathbf{E}^{2-3}\left(\sin ^{2} \xi^{2-4}-\sin ^{2} \xi^{1-3}\right)
\end{aligned}
$$




$$
\begin{aligned}
& +\frac{1}{4}\left(\mathbf{I}_{+}^{1-2}+\mathbf{I}_{-}^{1-2}\right)\left(\cos \xi^{1-3} \sin \xi^{1-3}-\cos \xi^{2-4} \sin \xi^{2-4}\right) \\
& +\frac{1}{4}\left(\mathbf{I}_{+}^{3-4}+\mathbf{I}_{-}^{3-4}\right)\left(\cos \xi^{1-3} \sin \xi^{1-3}-\cos \xi^{2-4} \sin \xi^{2-4}\right) \\
& -\frac{1}{4}\left(\mathbf{I}_{+}^{2-4}+\mathbf{I}_{-}^{2-4}\right)\left(\cos \xi^{1-3} \sin \xi^{1-3}+\cos \xi^{2-4} \sin \xi^{2-4}\right) \\
& -\frac{1}{4}\left(\mathbf{I}_{+}^{1-3}+\mathbf{I}_{-}^{1-3}\right)\left(\cos \xi^{1-3} \sin \xi^{1-3}+\cos \xi^{2-4} \sin \xi^{2-4}\right)
\end{aligned}
$$

The corrections do not change the conclusions of the paper. 